\title{
Mixing layer development in compound channel flows with submerged and emergent rigid vegetation over the floodplains
}

\author{
Victor Dupuis · Sébastien Proust - Céline Berni · André Paquier
}

Received: date / Accepted: date

\begin{abstract}
This laboratory study aims at investigating the longitudinal development of a mixing layer in a compound open-channel (two-stage geometry with a main channel and adjacent floodplains). The floodplains are covered with two roughness types: either a bed roughness representing a submerged dense meadow or emergent roughness elements (cylinders) representing an alluvial forest. The theoretical background used for plane mixing layers is adapted to the highly threedimensional mixing layer that develops at the main channel/floodplain interface. The mixing layer width is divided into two parts on either side of the interface. For the wooded floodplain, the mixing layer width on the floodplain side levels off downstream much more rapidly than for the grassed floodplain. The lateral profiles of normalised velocity and turbulence quantities are found to be self-similar in the longitudinal direction for a fixed elevation. However, shallowness effects prevented the normalised lateral profiles of velocity and turbulence quantities from coinciding at different elevations. The respective contributions of lateral Reynolds stresses and secondary currents to the lateral exchange of momentum are estimated. At the main channel/floodplain interface, the momentum exchange is driven by Reynolds stresses. In the main channel, both Reynolds stresses and secondary currents contribute to the lateral flux of momentum. Secondary cur-

V. Dupuis · S. Proust · C. Berni · A. Paquier

Irstea, UR HHLY Hydrologie-Hydraulique, Centre de Lyon-

Villeurbanne

5 rue de la Doua BP32108

F-69616 Villeurbanne cedex, France

Tel.: +33-47-2208787

Fax: +33-47-8477875

E-mail: victor.dupuis@centraliens-lille.org . bastien.proust@irstea.fr · celine.berni@irstea.fr . andre.paquier@irstea.fr
\end{abstract}

rents are stronger with emergent macro-roughness elements than with bed-roughness only on the floodplains. Large-scale turbulent coherent structures are investigated based on two-point space-time correlations of velocity. These structures are found to span the entire floodplain flow depth, and their convection velocity is close to the depth-averaged longitudinal velocity at the interface. The coherent fluctuations of the longitudinal and lateral velocities have different Strouhal number values, similar to those found in plane mixing layers.

Keywords Laboratory study - Compound channel . Turbulent mixing layer • Coherent structures . Secondary currents · Cylinder array

\section{Introduction}

During high flood events a river may overflow its banks. Then, a flow in a compound channel develops, corresponding to a two-stage geometry composed of the river's main channel and adjacent floodplains. The mixing layer generated by the difference in velocity between the deeper flow in the main channel and the shallower flow in the floodplain has three-dimensional features owing to the two-stage geometry and the vertical confinement of the flow (Knight and Shiono, 1990; Nezu and Nakayama, 1997). The present study aims at experimentally investigating the streamwise development towards longitudinal uniformity of the compound channel flow. This has scarcely been investigated so far. The theoretical background developed for plane and shallow mixing layers will be used and adapted for the compound channel mixing layer.

As experimentally shown by Bousmar et al (2005), the longitudinal development of the mean flow in a straight compound channel is a slow process, which can 
be attributed to the mixing layer development. Thus, the hypothesis of longitudinal uniformity for computing compound channel flows may not be applicable in most practical cases. The mixing layer between main channel and floodplain plays a major role in redistributing momentum within the river cross-section and in particular in accelerating the floodplain flow. A thorough comprehension of the mixing layer physical properties, of its spatial extension and of its longitudinal development is therefore an important point for computing compound channel flows.

To understand the specificity of the compound channel mixing layer, one should remember the characteristics of the plane and confined mixing layers in rectangular channels. The turbulent plane mixing layer is formed by two parallel streams of different velocities without vertical or lateral confinement of the flow. The width of the turbulent plane mixing layer grows linearly and indefinitely with increasing longitudinal distance from the generation point. The growth rate increases with the normalised velocity difference $\lambda=\left(U_{1}-U_{2}\right) /\left(U_{1}+U_{2}\right)$, where $U_{1}$ and $U_{2}$ are the velocities outside the mixing layer in the high speed stream and low speed stream, respectively (Yule, 1972; Brown and Roshko, 1974; Oster and Wygnanski, 1982). The growth rate is also sensitive to experimental conditions such as the thickness and state (laminar or turbulent) of the boundary layer at the trailing edge of the splitter plates (Bell and Mehta, 1990). It can also be influenced by forced periodic excitations near the splitter plates, caused e.g. by a resonance phenomenon in the experimental facility (Ho and Huerre, 1984). Except in the wake of the splitter plate, the flow in the turbulent plane mixing layer is self-similar, i.e. the lateral profiles of longitudinal mean velocity and of turbulence quantities are invariant in the longitudinal direction, when they are normalised by the velocity difference $U_{1}-U_{2}$, and when the lateral coordinate is normalised by the mixing layer width. Brown and Roshko (1974) showed from shadowgraphs that the velocity fluctuations of the plane turbulent mixing layer are driven by 2D horizontal coherent structures, whose shape and dynamics resemble the Kelvin vortex of the laminar mixing layer. These coherent structures are generated quasi-periodically.

Unlike the plane mixing layer, the shallow mixing layer is a vertically confined flow under the influence of both bed and free surface (Chu and Babarutsi, 1988; Uijttewaal and Booij, 2000). If the influence of the bed is sufficiently important, the mixing layer width levels off downstream. This stabilisation is attributed to the effect of the small-scale 3D bed-induced turbulence on the $2 \mathrm{D}$ horizontal coherent structures.
Mixing layers can also be generated by a lateral change in roughness across a flat channel. Such flows were investigated by Vermaas et al (2011) in the case of a rough bed adjacent to a smooth bed, and by White and Nepf (2007) and Zong and Nepf (2010) in the case of an array of emergent cylinders adjacent to a smooth bed. Vermaas et al (2011) showed that in the fully developed flow region, the contribution of secondary currents to the lateral exchange of longitudinal momentum can exceed that of the lateral Reynolds stresses. This makes an important difference with the plane mixing layer for which longitudinal vorticity, although present, plays a rather minor role in the lateral momentum exchange (Bernal and Roshko, 1986). White and Nepf (2007) and Zong and Nepf (2010) showed that, in a similar way to shallowness, the presence of emergent roughness elements on one side of the mixing layer makes the mixing layer width stabilise downstream. This is attributed to the high energy dissipation of the coherent structures in the cylinder array. They found that the elementary coherent structure is quasi-2D and consists in a Kelvin-type vortex associated with a strong ejection event (transfer of slow-moving fluid into the high speed region) upstream of the vortex and a somewhat weaker aspiration event (transfer of fast-moving fluid into the low speed region) downstream of the vortex. The lateral flux of longitudinal momentum is mostly due to the ejection and aspiration events, while the contribution to this flux by the secondary currents is one order of magnitude lower.

For practical reasons, plane and shallow mixing layers are always laterally confined in laboratory experiments. This results in a reduction of the velocity difference $U_{1}-U_{2}$ and in a lateral homogenisation of the flow when going downstream (Uijttewaal and Booij, 2000). Furthermore, Wood and Bradshaw (1984) showed that the presence of a side wall influences the turbulence budget within the plane mixing layer, both before and after the mixing layer reaches the wall.

Compared to the previous types of mixing layer, the compound channel mixing layer has specific properties that make the physical processes somewhat different (e.g. Shiono and Knight, 1991; Proust et al, 2013). The difference in flow depth and bed roughness between the two subsections (main channel and floodplain) induces an asymmetric mixing layer with respect to the main channel/floodplain interface. The lateral distribution of longitudinal velocity cannot therefore be represented with an antisymmetric function such as hyperbolic tangent or error functions as proposed for plane mixing layers. The friction on the bed induces a vertical boundary layer that interacts with the mixing layer and makes it three-dimensional, similarly to shallow mixing layers. 
Moreover, the compound channel mixing layer is laterally confined, either by side walls or by the mixing layer on the opposite floodplain. But contrary to plane and shallow mixing layers, the lateral flow confinement does not induce a reduction of the velocity difference $U_{1}-U_{2}$ when going downstream, as the velocity difference is self-sustained by the two-stage geometry.

According to Knight and Demetriou (1983), Knight and Shiono (1990) and Tominaga and Nezu (1991), the main driving parameters of a straight compound channel flow are: the relative flow depth $D_{r}=H_{f} / H_{m}$ (ratio of the flow depth in the floodplain $H_{f}$ to that in the main channel $H_{m}$ ); the width-to-depth-ratios of main channel and floodplain; the ratio of main channel width to floodplain width; the roughness in each subsection; and, for trapezoidal subsections, the bank slopes of the main channel and/or floodplain. The decrease in relative depth $D_{r}$ or the increase in the difference in roughness between floodplain and main channel both result in increasing the velocity difference across the mixing layer and therefore in enhancing turbulence production (Fernandes, 2013). Relative depth, width-todepth-ratios and bank slopes of each subsection largely determine the secondary currents (Tominaga and Nezu, 1991; Blanckaert et al, 2010), which in turn play an important role in the momentum distribution across the compound cross-section (Kara et al, 2012). For the same width-to-depth-ratio in the main channel and the same relative flow depth, symmetric (two identical floodplains at both sides of the main channel) and asymmetric (one floodplain at one side and a vertical wall at the other side) compound sections lead to different flow fields (Proust et al, 2013). In symmetrical layout the two opposite mixing layers at both sides of the main channel can meet at the main channel centreline. In asymmetrical layout, the mixing layer can reach the lateral boundary layer of the vertical wall.

Compound channel flows were investigated in previous laboratory studies for different types of floodplain roughness: smooth bed (Knight and Shiono, 1990; Stocchino and Brocchini, 2010; Peltier, 2011), rough bed (Nicollet and Uan, 1979; Tominaga and Nezu, 1991; Fernandes, 2013) and emergent macro-roughness elements (Pasche and Rouvé, 1985; Kozioł, 2013). The flow is generally analysed at a constant downstream position, where it is considered as fully developed. Only few studies considered the longitudinal flow development. Proust et al (accepted) in the case of a smooth compound channel and Fernandes (2013) in the case of a compound channel with rough floodplains showed that the mixing layer width stabilises downstream and that the initial growth rate is comparable to that of a plane mixing layer.
Sellin (1964) already identified large-scale horizontal coherent structures in compound channel flows. Stocchino and Brocchini (2010) showed that macrovortices at the main channel/floodplain interface are only present for sufficiently shallow flows $\left(D_{r}<0.5\right)$. According to Proust et al (accepted), large coherent structures can only be present for $\lambda^{\prime}>0.3$, where $\lambda^{\prime}=$ $\left(U_{d 1}-U_{d 2}\right) /\left(U_{d 1}+U_{d 2}\right)$ is based on the depth-averaged velocities (subscript $d$ refers to depth-averaging). Tamai et al (1986) showed that, despite the complex 3D organisation of the coherent structures in compound channel, their periodicity can be predicted by a 2D stability analysis.

The present experimental study investigates the longitudinal development of the compound channel mixing layer for two flow configurations, with either a bed roughness or an emergent cylinder array on the floodplain. The flow conditions are such that $D_{r}<0.5$ and $\lambda^{\prime}>0.3$, therefore horizontal coherent structures are present in the mixing layer and are investigated.

The experimental setup and the flow conditions of the two test cases investigated are described in Section 2. The longitudinal development of the flows is analysed in Section 3. Then, the vertical heterogeneity of the compound channel mixing layer is discussed in Section 4 . The effects of secondary currents and lateral Reynolds stresses on the cross-sectional distribution of momentum and on the force balance are presented in Section 5. Finally, the coherent structures associated with the mixing layer are investigated in Section 6 .

\section{Experimental setup and methodology}

The experiments were performed in an $18 \mathrm{~m}$ long and $3 \mathrm{~m}$ wide glassed-wall flume, located in the Hydraulics and Hydromorphology Laboratory of Irstea, LyonVilleurbanne, France. The compound channel crosssection was symmetrical and composed of a rectangular central main channel of width $B_{m}=1 \mathrm{~m}$ and of two adjacent floodplains of width $B_{f}=1 \mathrm{~m}$ (see crosssectional view in Fig. 1). The longitudinal bottom slope was $S_{0}=1.05 \mathrm{~mm} . \mathrm{m}^{-1}$. Both right and left floodplains were covered with dense plastic grass, whose $5 \mathrm{~mm}$-long blades were rigid. The main channel bankfull level, from the bottom of the main channel to the crest of the grass blades, was $z_{B F}=115 \mathrm{~mm}$.

The $x_{-}, y$ - and $z$-axes refer to the longitudinal (along flume bottom), lateral and vertical (normal to flume bottom) directions, respectively. In this coordinate system, the instantaneous velocities, timeaveraged velocities and velocity fluctuations are denoted $(u, v, w),(U, V, W)$ and $\left(u^{\prime}, v^{\prime}, w^{\prime}\right)$. Overline denotes time-averaging (e.g. $\left.\overline{u^{\prime} v^{\prime}}\right)$. The origin of the lon- 


\begin{tabular}{|c|c|c|c|c|}
\hline (1) & (2) & & Left floodplain & (3) \\
\hline (4)咅 & (3) & 2 & Main channel & \\
\hline & & $\mathrm{y}$ & Right floodplain & \\
\hline
\end{tabular}

(1) Inlet tanks

(2) Floodplain inlet ramps

(3) Splitter plates

(4) Honeycomb

(5) Downstream weirs

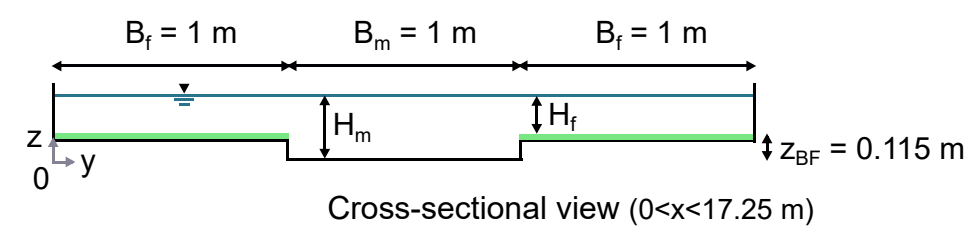

Fig. 1: Plan view and cross-sectional view (constant for $0<x<17.25 \mathrm{~m}$ ) of the compound channel flume.

gitudinal coordinate is defined at the trailing edge of the upstream splitter plates (see below). The origin of the lateral axis is located at the side wall of the right floodplain. The origin of the vertical coordinate is defined at the bottom of the main channel. In addition, a relative vertical coordinate $z_{f}$ is defined from the floodplain bottom (bankfull level of the main channel), i.e. $z_{f}=z-z_{B F}$.

The inlet discharge in each of the three subsections (main channel, right and left floodplains) was regulated independently with a control valve and an electromagnetic discharge-meter. The standard deviation of the discharge time series was of the order of $1.5 \%$ of the mean discharge value. After leaving the inlet tank, the flows in the right and left floodplains were accelerated along a ramp which elevation increases linearly from $z=0 \mathrm{~mm}$ to $z=115 \mathrm{~mm}$ between $x=-0.75 \mathrm{~m}$ and $x=0 \mathrm{~m}$ (see plan view in Fig. 1 ). The streams in the three subsections were separated by the vertical splitter plates until the ramp end at $x=0$. Water injection in the three inlet tanks is located below the main channel bottom level and oriented downwards. A $100 \mathrm{~mm}$-thick honeycomb ( $8 \mathrm{~mm}$ alveolus) was installed in the inlet tank of the main channel to vertically and laterally homogenise the flow. Thus, the velocity and turbulence fields are as homogeneous as possible at $x=0 \mathrm{~m}$. At the flume outlet, the flow was controlled by three independent weirs (one per subsection). Downstream splitter plates maintained the flow in the three subsections separated over a distance of $50 \mathrm{~cm}$ upstream of the weirs.

Two flow test cases were investigated, with floodplains covered either with plastic grass only or with an array of emergent cylinders set on the plastic grass. These two roughness types represent a submerged meadow and a woodland with emergent trees on the floodplain, respectively. These two test cases are termed CM (for Compound channel flow with Meadow over the floodplains) and CW (for Compound channel flow with Wood on the floodplains). The total discharge was the same in the two test cases $\left(Q_{t o t}=162 \mathrm{~L} . \mathrm{s}^{-1}\right)$. Following Bousmar et al (2005) the upstream discharge distribution and the downstream weir levels were adjusted by an iterative process in order to get a free surface parallel to the channel bed and to minimize the lateral net mass exchange between subsections. The flow conditions are reported in Table 1 . Subscripts $f$ and $m$ refer to the floodplain and the main channel flow respectively.

The cylinder array for test case CW was made of wooden circular cylinders that were uniformly distributed in staggered rows (see Fig. 2b). The cylinder diameter was $D=10 \mathrm{~mm}$ and the cylinder density $N=81$ cylinders. $\mathrm{m}^{-2}$. They were held together by an emergent wooden superstructure (see Fig. 2a). The accuracy of each cylinder position was $\pm 5 \mathrm{~mm}$ in both lateral and longitudinal directions.

The free surface levels were measured with ultrasonic sensors (UNDK20I69, Baumer) with an accuracy of $\pm 0.5 \mathrm{~mm}$. Longitudinal profiles of free surface level were measured at 7 lateral positions $(y=$ $300,700,1200,1500,1800,2200,2700 \mathrm{~mm}$ ) with a longitudinal step of $1 \mathrm{~m}$. Each measurement was obtained by averaging time series at $50 \mathrm{~Hz}$ over $3 \mathrm{~min}$. In each subsection and for both flows, the average water depth was found to be constant between $x=2$ and $x=17 \mathrm{~m}$, with discrepancies of $\pm 1 \mathrm{~mm}$. Two types of velocity measurements were carried out: single-point measurements with a unique ADV probe and two-point space-time correlation measurements with two ADV probes. Side-looking ADV probes were used (Vectrino Plus, Nortek), working with a sampling rate of $100 \mathrm{~Hz}$. The accuracy of the 
Table 1: Flow conditions of the two test cases: total discharge $Q_{t o t}$, discharge in the main channel $Q_{m}$, discharge in each of the two floodplains $Q_{f}$, water depth in the main channel $H_{m}$, relative flow depth $D_{r}$, Reynolds number

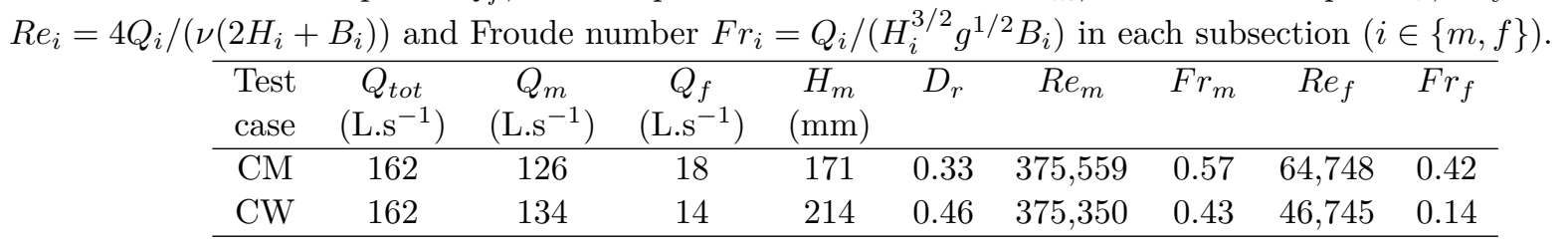

(a)

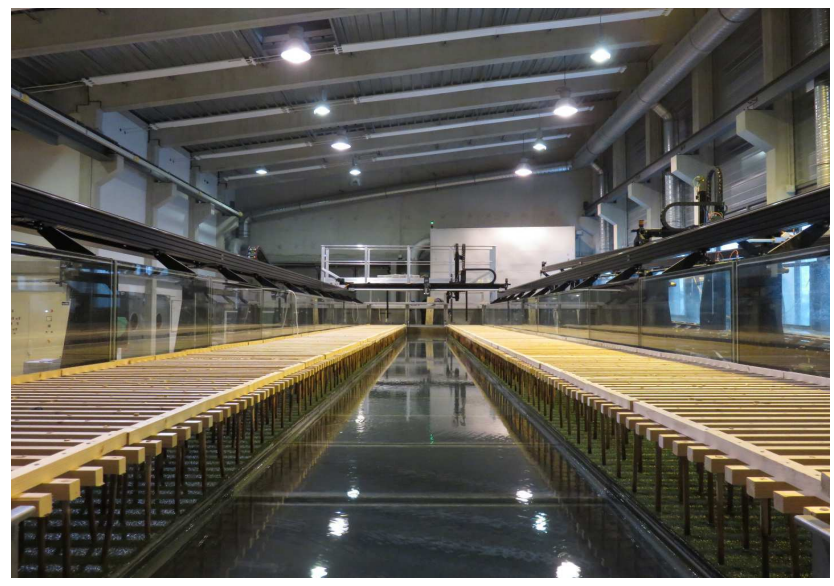

(b)

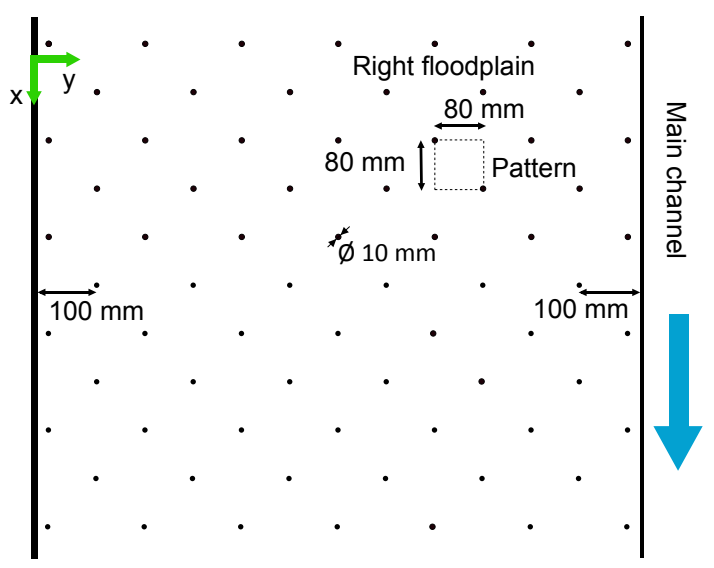

Fig. 2: (a) Picture from downstream of the compound channel flume located at Irstea, with wooden cylinders set on plastic grass over the floodplain (test case CW). (b) Plan view of the cylinder array arrangement for test case CW.

$\mathrm{ADV}$ was $\pm 0.5 \%$ of the measured value for the mean velocities and $\pm 1 \%$ for the Reynolds stresses. A recording time of $120 \mathrm{~s}$ was found to be sufficient to obtain mean velocities converged at $\pm 0.2 \mathrm{~cm} . \mathrm{s}^{-1}$ and Reynolds stresses converged at $\pm 0.2 \mathrm{~Pa}$. The recording time was increased to $600 \mathrm{~s}$ for autocorrelations and spatial correlation measurements. The ADV raw data were filtered with the software WinADV based on the despiking concept developed by Goring and Nikora (2002).

Velocity fields in the $(y, z)$-plane were measured across the compound section at five $x$-stations along the flume for test case CM and at four $x$-stations, but only in the main channel, for test case $\mathrm{CW}$ (indeed the high flow heterogeneity in the wooded floodplain requires very refined measuring mesh). Figure 3 illustrates the measuring mesh used for test case CM at $x=15.05 \mathrm{~m}$, consisting in 597 points. The lateral mesh step ranged from $10 \mathrm{~mm}$ (near the interface) to $100 \mathrm{~mm}$ (outside the mixing layer) and the vertical mesh step ranged from $4 \mathrm{~mm}$ to $15 \mathrm{~mm}$. Vertical profiles in the main channel were composed of 20 measuring points for test case $\mathrm{CM}$ and 24 points for test case CW.

The main channel discharge $Q_{m}$ was calculated by integrating the velocity field over the main channel cross-section for each $x$-station. The maximum discrep-

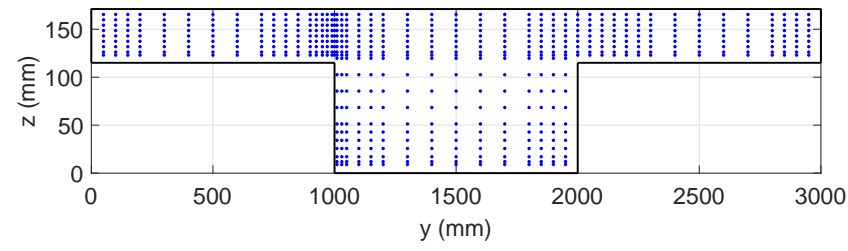

Fig. 3: Cross-sectional measuring mesh used for ADV measurements (test case CM).

ancy between the inlet main channel discharge (measured with the discharge-meter) and the discharge obtained by velocity integration at the different $x$-stations was $1.1 \%$ for test case $\mathrm{CM}$ and $0.9 \%$ for test case $\mathrm{CW}$. The discharge distribution between subsections was thus considered to be constant along the channel for both test cases.

Given the symmetry of the compound cross-section, we mostly present measurements across the right-hand half of the flume $(0<y<1500 \mathrm{~mm})$. The vertical $(x, z)$-plane at the main channel/floodplain boundary is called interface and the right-hand interface position is denoted $y_{\text {int }}\left(y_{\text {int }}=1000 \mathrm{~mm}\right)$. 


\section{Longitudinal flow development}

3.1 Cross-sectional distribution of flow quantities

Figure 4 shows cross-sections of mean longitudinal velocity $U(y, z)$ (total cross-section) and lateral turbulence intensity $\rho \overline{v^{\prime 2}}(y, z)(500<y<1500)$ at different $x$-stations for test case CM. The flow is fairly symmetrical about the main channel centreline. The distribution of both $U$ and $\rho \overline{v^{\prime 2}}$ is evolving when going downstream until becoming fairly constant from $x=13.15 \mathrm{~m}$. The maximum velocity is located beneath the free surface and progressively moves upwards when going downstream, from $z \approx 60 \mathrm{~mm}$ at $x=3.35 \mathrm{~m}$ to $z \approx 115 \mathrm{~mm}$ at $x=13.15 \mathrm{~m}$. As will be shown in Section 5.1, secondary currents are mostly responsible for the location of the $U$-maximum. The turbulence level in the dark blue region at $x=3.35 \mathrm{~m}$ (Fig. 4f) is $\sqrt{\overline{u^{\prime 2}}} / U=0.036$, indicating that the flow is weakly turbulent when leaving the inlet tanks. The main source of turbulence in the channel is the mixing layer, which creates a region of high lateral turbulence intensity around $y=1000 \mathrm{~mm}$. This turbulence region expands on either side of the interface when going downstream. Another source of turbulence is the boundary layer induced by the bottom and the side walls of the main channel and this turbulence diffuses towards the free surface when going downstream.

Figure 5 shows $U(y, z)$ and $\rho \overline{v^{\prime 2}}(y, z)$ for test case $\mathrm{CW}$, but only in the right-hand half cross-section of the main channel. The observations are similar to test case CM (upward displacement of the region of maximum velocity, lateral extension of the turbulence region at the interface, turbulence production at the main channel bottom).

The distributions of $U$ and $\rho \overline{v^{\prime 2}}$ are very similar in the three last measuring stations for both flows (this was also observed for the other turbulence quantities). An analysis of the vertical profiles of $U, \overline{u^{\prime 2}}, \overline{v^{\prime 2}}$ and $\overline{u^{\prime} v^{\prime}}$ at the interface (not shown here, see Dupuis, 2016) shows that the development towards a constant value is faster for test case CW than for test case CM, all these flow quantities being constant at the interface beyond $x \approx 6 \mathrm{~m}$ and $x \approx 11 \mathrm{~m}$, respectively. However, the seeming longitudinal uniformity of the flow field, inferred from a visual observation of Figs. 4 and 5, will be invalidated in the next section when analysing the mixing layer properties.
3.2 Mixing layer

\subsubsection{Inflection point position}

For plane mixing layers, $U(y)$ is antisymmetric (of hyperbolic tangent type). Thus the centre of the mixing layer can be equally defined as (i) the geometrical centre of the mixing layer, (ii) the lateral position $y_{U_{0}}$ where the velocity $U_{0}=\left(U_{1}+U_{2}\right) / 2$ is reached, and (iii) the lateral position $y_{I P}$ of the inflection point in $U(y)$. In compound channels, these three positions do not necessarily collapse (see sketch in Fig. 6a). As the inflection point is the primary source of flow instability (Fjortoft, 1950), we consider here the inflection point position as the centre of the mixing layer. The position of the inflection point $y_{I P}$ has been observed to be constant over the depth and whole length of the flume, and is located very close to the interface, at $y_{I P}=1000 \pm 5 \mathrm{~mm}$ for both test cases $\mathrm{CM}$ and $\mathrm{CW}$. The position of $y_{I P}$ is thus essentially driven by the position of the vertical bank, i.e. by the topographical forcing caused by the two-stage geometry.

\subsubsection{Mixing layer width}

The mixing layer width was estimated at different $x$ positions at a constant altitude $z_{f}=H_{f} / 2$. To take into account the asymmetry of the compound channel mixing layer with respect to the interface, two mixing layer widths on either side of the interface are defined: the main channel mixing layer width $\delta_{m}$ and the floodplain mixing layer width $\delta_{f}$ (see sketch in Fig. 6a), such that :

$U\left(y_{I P}+\delta_{m} / 2\right)=\frac{U_{1}+U_{I P}}{2}$

$U\left(y_{I P}-\delta_{f} / 2\right)=\frac{U_{2}+U_{I P}}{2}$

where $U_{1}$ is the maximum velocity in the main channel, $U_{2}$ is the velocity in the floodplain outside the mixing layer in the plateau region and $U_{I P}$ is the velocity at the inflection point. These definitions are consistent with the choice of $y_{I P}$ as the mixing layer centre. Note that for an antisymmetric mixing layer $\left(U_{I P}=U_{0}\right)$, the total mixing layer width $\delta_{t o t}=\delta_{m}+\delta_{f}$ is the same as the one defined by Van Prooijen et al (2005). In the case of a mixing layer induced by a cylinder array adjacent to a smooth bed, White and Nepf (2007) also defined two independent length scales on the high- and low-speed sides of the mixing layer.

Figure $6 \mathrm{~b}$ shows the longitudinal evolution of widths $\delta_{m}$ and $\delta_{f}$. The measurement accuracy of $\delta_{i}$, evaluated by repeating the measurements, was better than 

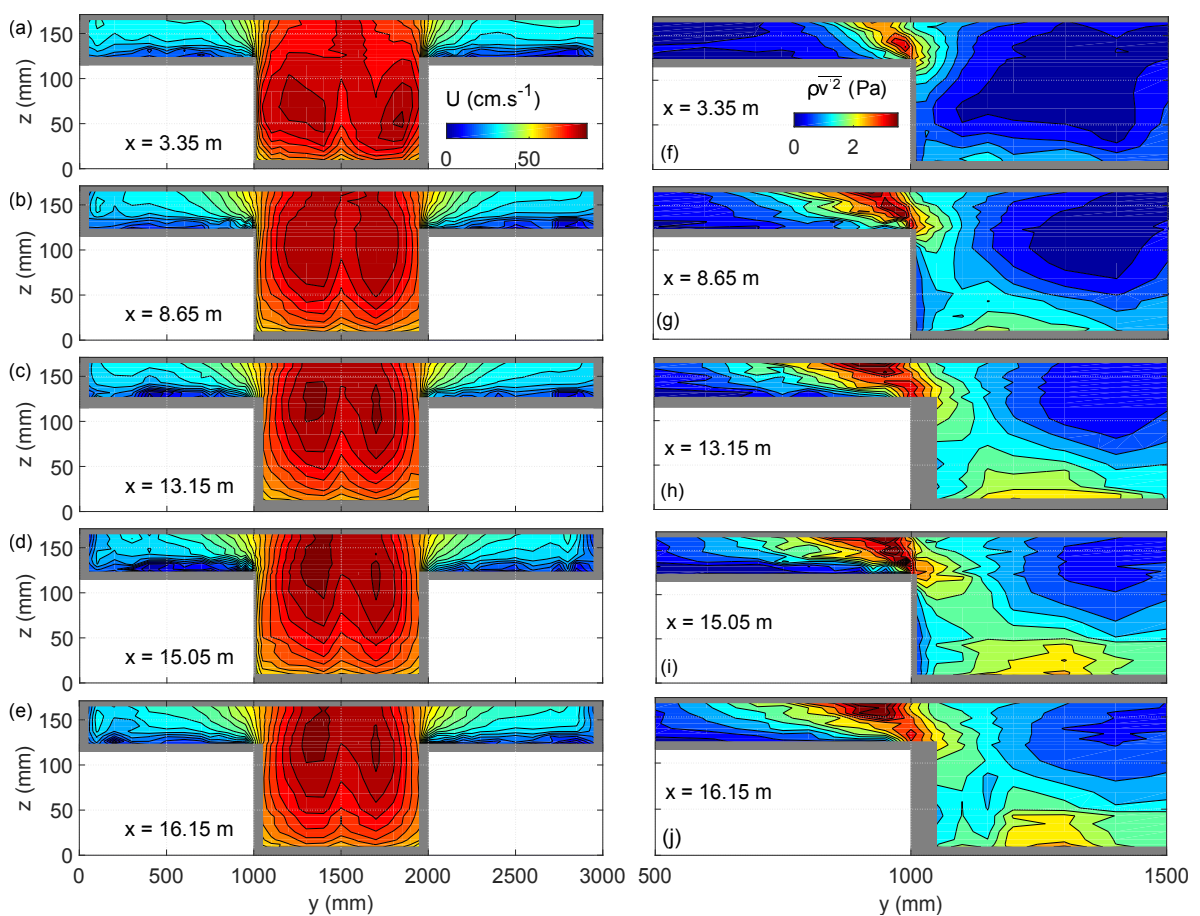

Fig. 4: Development towards longitudinal uniformity of a compound channel flow with plastic grass over the floodplains (test case CM).(a-e) Mean longitudinal velocity $U$ across the total compound cross-section and (f-j) lateral turbulence intensity $\rho \overline{v^{\prime 2}}$ in the right-hand half cross-section at various $x$-positions. Regions where measurements are unavailable are shaded grey.

$\pm 5 \mathrm{~mm}$. The main channel mixing layer width $\delta_{m}$ increases all along the flume for both test cases. Width $\delta_{f}$ levels off at $x \approx 5 \mathrm{~m}$ in the wooded floodplain $(\mathrm{CW})$; the observed scatter can be attributed to local variations in bed topography or cylinder position. For test case CM, only the two last values of $\delta_{f}$ are close to each other. Given that these two measurements are sufficiently away from the channel outlet for not being influenced by the end conditions, it can be inferred that $\delta_{f}$ has probably stabilised at $x \approx 13 \mathrm{~m}$ for test case CM. The growth rate in the main channel is similar for both test cases $\left(\partial \delta_{m} / \partial x \approx 10 \mathrm{~mm} . \mathrm{m}^{-1}\right)$ and is lower than that observed on the floodplain side for test case CM $\left(\partial \delta_{f} / \partial x \approx 14 \mathrm{~mm} \cdot \mathrm{m}^{-1}\right)$.

Previous studies highlighted three effects that can induce a stabilisation of the mixing layer width: vertical flow confinement (Chu and Babarutsi, 1988), lateral flow confinement (Wood and Bradshaw, 1984) and the presence of emergent roughness elements, like a cylinder array (White and Nepf, 2007). In the present experiments, the smooth main channel is too wide and the flow too deep compared with the length of the flume to observe a stabilisation of the mixing layer width. In many compound channel experiments presented in the literature, the main channel is much nar- rower than in the present case (Peltier, 2011; Stocchino and Brocchini, 2010). Thus lateral confinement becomes significant and the mixing layer is constrained more rapidly when developing downstream, either by the lateral boundary layer in the case of an asymmetrical compound channel or by the opposite mixing layer in the case of a symmetrical compound channel. Fernandes (2013) observed that the mixing layer width on the main channel side was the same for smooth and rough floodplains for identical relative depth $D_{r}$; this suggests that the dynamics of $\delta_{m}$ is weakly influenced by the floodplain roughness but is mostly driven by the main channel geometry.

The likely stabilisation of $\delta_{f}$ in the grassed floodplain can be explained by vertical confinement. Chu and Babarutsi (1988) normalised the stabilisation distance of the shallow mixing layer width $x$ in the form $x^{*}=x c_{f} / H$, where $H$ is the water depth and $c_{f}$ is the average bed friction coefficient across the mixing layer $\left(c_{f}=0.5\left(c_{f 1}+c_{f 2}\right)\right.$ with $c_{f i}=\tau_{b} /\left(0.5 \rho U_{i}^{2}\right)$, $i \in\{1,2\}$ and $\tau_{b}$ the bed shear stress). They found that the shallow mixing layer reaches a constant width at $x^{*} \approx 0.6-1$. Considering only the floodplain part of the mixing layer, we can define $x_{f}^{*}=x c_{f, f} / H_{f}$, where $c_{f, f}=\tau_{b} /\left(0.5 \rho U_{f}^{2}\right)$. We then obtain $x_{f}^{*}=1.6$ 

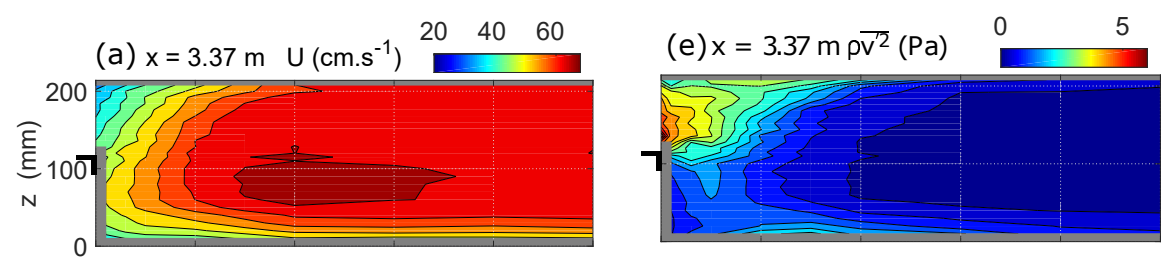

(b) $x=8.66 \mathrm{~m}$

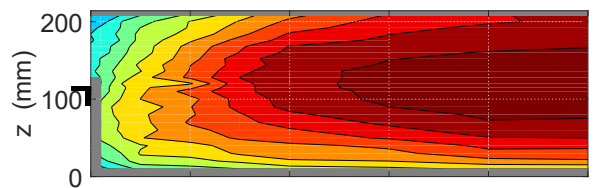

(f) $x=8.66 \mathrm{~m}$

(c) $x=12.99 \mathrm{~m}$
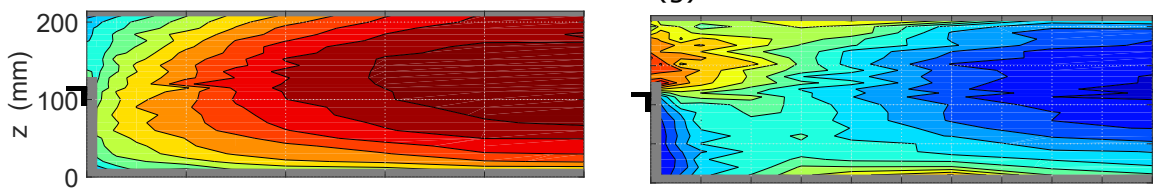

(d) $x=15.08 \mathrm{~m}$

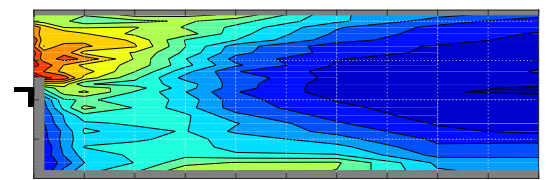

(g) $x=12.99 \mathrm{~m}$

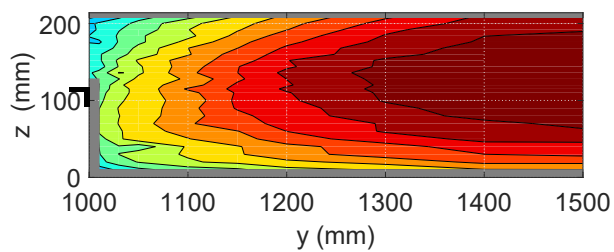

(h) $x=15.08 \mathrm{~m}$

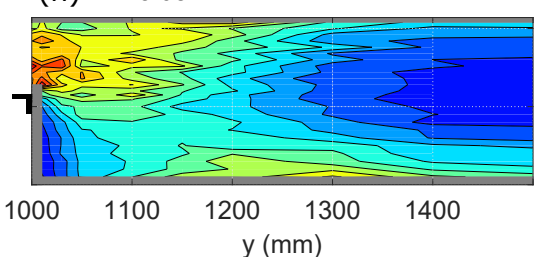

Fig. 5: Development towards longitudinal uniformity of a compound channel flow with an emergent cylinder array on the floodplains (test case CW). (a-d) Mean longitudinal velocity $U$ and (e-h) lateral turbulence intensity $\rho \overline{v^{\prime 2}}$ in the right-hand half cross-section of the main channel at various $x$-positions. Regions where measurements are unavailable are shaded grey. The black edge on the vertical axis indicates the bankfull level of the main channel.
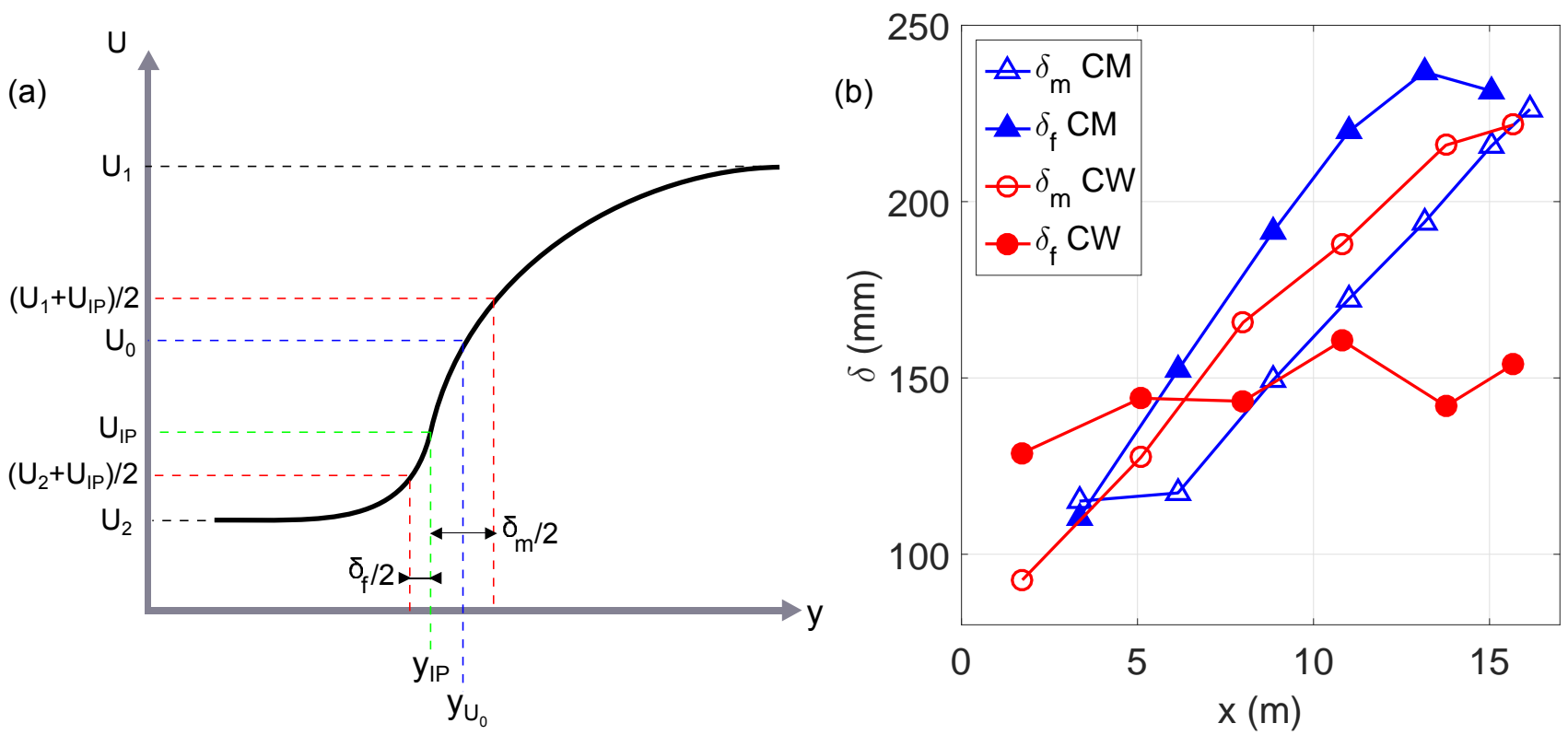

Fig. 6: (a) Definition of the main channel and floodplain mixing layer widths, $\delta_{m}$ and $\delta_{f}$, calculated from the lateral profile of mean longitudinal velocity $U$. (b) Longitudinal variation in $\delta_{m}$ and $\delta_{f}$ at $z_{f} / H_{f}=0.5$. 
$(x \approx 13 \mathrm{~m})$ for test case CM, which is of the same order of value suggested by Chu and Babarutsi (1988).

The rapid stabilisation of $\delta_{f}$ for test case CW can be related to the presence of the cylinder array. According to White and Nepf (2007) and Zong and Nepf (2010), the lateral penetration of the mixing layer and of the associated coherent structures into a cylinder array is limited by the strong energy dissipation due to the cylinder drag.

It can be concluded that none of the two flows reached longitudinal uniformity at the downstream end of the channel, unlike the first visual impression from observing Figs. 4 and 5. A refined investigation of the mixing layer shows that the total mixing layer width $\delta_{t o t}=\delta_{m}+\delta_{f}$ always increases along the flume.

\subsubsection{Self-similar flow development}

The theory of plane mixing layer (Yule, 1972) states that the lateral profiles of mean velocity and turbulence quantities are self-similar in their longitudinal development, except in the near-field region, where the flow is still influenced by the splitter plate wake. We therefore decided to investigate the likely self-similarity of the compound channel mixing layer. Lateral profiles $U(y), \sqrt{\overline{u^{\prime 2}}}(y)$ and $\overline{u^{\prime} v^{\prime}}(y)$ are plotted in Fig. 7 for both test cases at a constant elevation $z_{f}=H_{f} / 2$. The three quantities are normalised by the velocity difference $U_{1}-U_{2}$, similarly to plane mixing layers. To take into account the asymmetry of the mixing layer, the lateral coordinate $y$ is normalised by $\delta_{f}$ in the floodplain and by $\delta_{m}$ in the main channel. Apart from the most upstream profile (blue '+') that may still be influenced by the upstream boundary conditions, the mixing layer is found to be self-similar for both test cases.

Table 2 shows the normalised peak values of $\sqrt{\overline{u^{\prime 2}}}$, $\sqrt{\overline{\overline{v^{\prime 2}}}}$ and $\sqrt{\overline{\overline{u^{\prime} v^{\prime}}}}$ measured at $z_{f}=H_{f} / 2$ for test cases $\mathrm{CM}$ and $\mathrm{CW}$, compared with typical values for plane mixing layers (Olsen and Dutton, 2002) and with values for shallow mixing layers (Uijttewaal and Booij, 2000). For test case $\mathrm{CW}$, the peak values are measured $120 \mathrm{~mm}$ downstream of the cylinder positioned at $y=980 \mathrm{~mm}$, such that the peak values are not due to the cylinder wake. Some higher values of $\sqrt{\overline{u^{\prime 2}}}$ max $/\left(U_{1}-U_{2}\right)$ and $\sqrt{\overline{u^{\prime} v^{\prime}}}$ max $/\left(U_{1}-U_{2}\right)$ are observed for test case CW but globally the peak turbulence values for plane, shallow and compound channel mixing layers are of the same order of magnitude.
Table 2: Normalised peak values of $\sqrt{\overline{\overline{u^{\prime 2}}}}, \sqrt{\overline{\overline{v^{\prime 2}}}}$ and $\sqrt{\overline{u^{\prime} v^{\prime}}}$ for the compound channel test cases CM and CW at $z_{f}=H_{f} / 2$, for the plane mixing layer (PML, from Olsen and Dutton, 2002) and for the shallow mixing layer (SML, from Uijttewaal and Booij, 2000).

\begin{tabular}{cccc}
\hline & $\frac{\left(\sqrt{\overline{u^{\prime 2}}}\right)_{\max }}{U_{1}-U_{2}}$ & $\frac{\left(\sqrt{\overline{v^{\prime 2}}}\right)_{\max }}{U_{1}-U_{2}}$ & $\left(\sqrt{\overline{u^{\prime} v^{\prime}}}\right)_{\max }$ \\
& & & \\
\hline$U_{1}-U_{2}$ & \\
CM & 0.17 & 0.11 & 0.10 \\
CW & 0.21 & 0.13 & 0.13 \\
PML & 0.17 & 0.14 & 0.10 \\
SML & 0.13 & 0.11 & 0.09 \\
\hline
\end{tabular}

\section{Vertical heterogeneity of the mixing layer}

\subsection{Mixing layer widths and $\lambda$-ratio}

Vertical profiles $\delta_{m}(z)$ and $\delta_{f}(z)$ at $x=15.05 \mathrm{~m}$ for test case CM and at $x=11.76 \mathrm{~m}$ for test case CM are presented in Fig. 8a. Both widths $\delta_{m}$ and $\delta_{f}$ increase when going towards the free surface. However, the increase in $\delta_{f}$ in the wooded floodplain $(\mathrm{CW})$ is less significant than for $\delta_{f}$ in the grassed floodplain $(\mathrm{CM})$ and for $\delta_{m}$ for both roughness types. This is related to the vertical homogenisation of the flow exerted by the cylinder array (Liu et al, 2008; Dupuis et al, 2016).

At constant elevation $z_{f} / H_{f}=0.5$, the normalised velocity difference $\lambda=\left(U_{1}-U_{2}\right) /\left(U_{1}+U_{2}\right)$ approximately increases from 0.40 to 0.50 for test $\mathrm{CM}$ and from 0.70 to 0.74 for test case $\mathrm{CW}$ between $x=2$ and $x=15 \mathrm{~m}$ (not shown). This longitudinal increase in $\lambda$ is due to the increase in the maximum velocity in the main channel $U_{1}$, whereas $U_{2}$ keeps nearly constant at fixed elevation. Vertical profiles $\lambda(z)$ are shown in Fig. 8b. For both test cases, a strong increase is observed near the floodplain bottom $z_{f} / H_{f}=0$, which is due to the decrease in $U_{2}$ near the floodplain bottom. Similarly to $\delta_{f}$, variations of $\lambda$ along the vertical are larger for test case CM than for test case CW.

The compound channel mixing layer is therefore very heterogeneous in the vertical direction. However, the presence of an emergent cylinder array makes the mixing layer more two-dimensional.

4.2 Lateral profiles of mean velocity and turbulence quantities

Lateral profiles $U(y), \overline{u^{\prime} v^{\prime}}(y), \sqrt{\overline{u^{\prime 2}}}(y)$ and $\sqrt{\overline{v^{\prime 2}}}(y)$ at $x=13.15 \mathrm{~m}$ and at three different elevations $z_{f} / H_{f}=$ $0.27,0.50$ and 0.89 , are presented in Fig. 9 for test case 

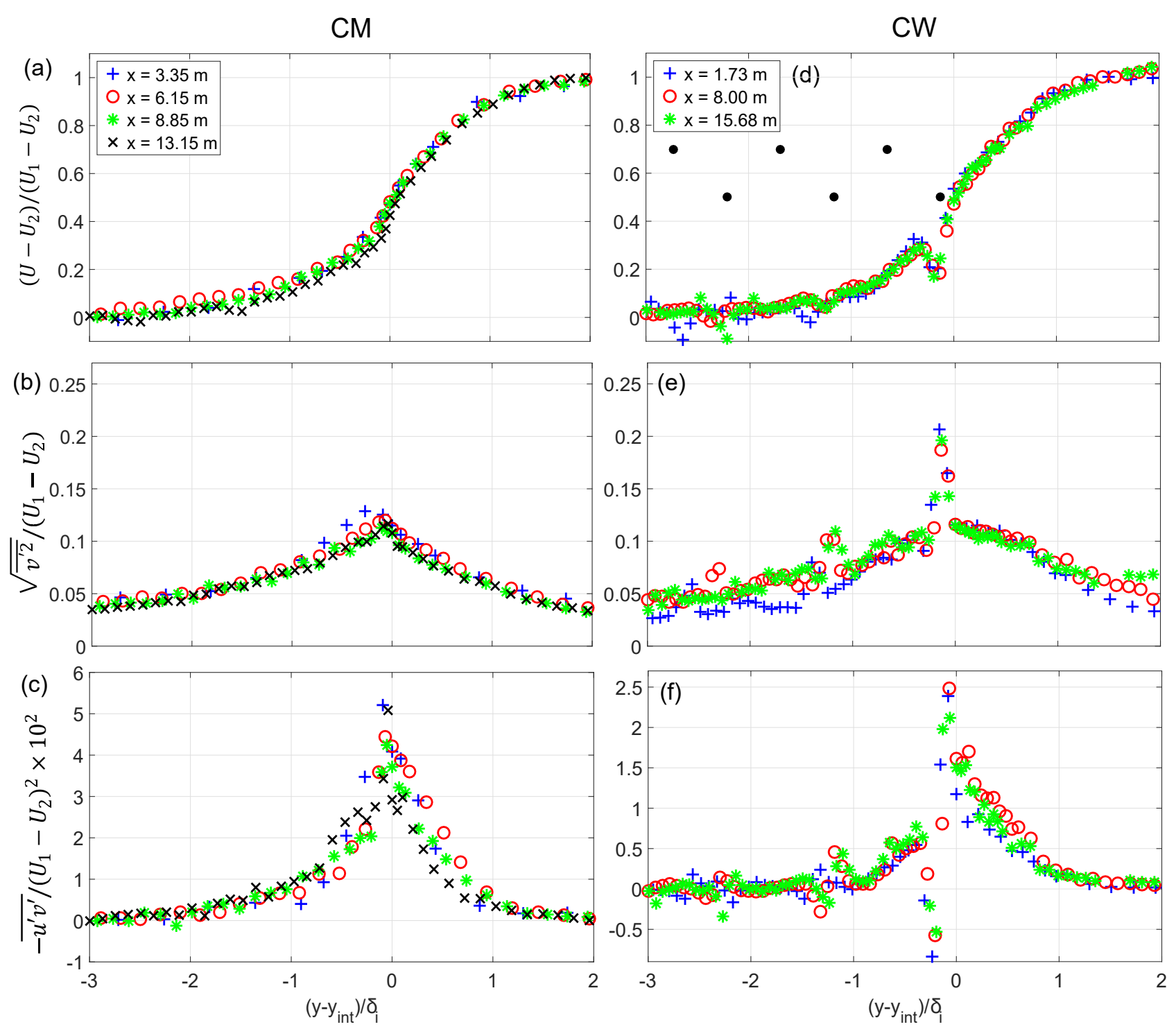

Fig. 7: Lateral profiles at elevation $z_{f}=H_{f} / 2$ of normalised longitudinal mean velocity, lateral turbulence intensity and lateral Reynolds stress for (a-c) test case CM and (d-f) test case CW. The mixing layer width $\delta_{i}$ is equal to $\delta_{f}$ on the floodplain side and to $\delta_{m}$ on the main channel side. For test case CW, the measurement is located downstream of a lateral row of cylinders with seven cylinders (Fig. 2). Black dots in chart d show the cylinder positions.

CM (study not performed for test case CW). These lateral profiles have been normalised in the same way as in Fig. 7, widths $\delta_{i}$ and velocities $U_{1}$ and $U_{2}$ being calculated at the considered elevation. The profiles do not coincide over the depth, particularly on the floodplain side $\left(y-y_{I P}\right) / \delta_{i}<0$. The magnitudes of the peak turbulence quantities vary across the water column, as well as the position of the peaks. These vertical variations can be attributed to the shallowness effect in the floodplain: due to bed-induced turbulence, the turbulence budget and flow equilibrium are necessarily different in the near bed region and in the free-surface region. On the main channel side, where shallowness effects are lower, the differences between the normalised profiles are less important.

On the floodplain side, in the near interface region $-1<\left(y-y_{I P}\right) / \delta_{i}<0$, a different behaviour can be observed between $\sqrt{\overline{u^{\prime 2}}} /\left(U_{1}-U_{2}\right)$ on the one hand, which increases when approaching the bed (from $z_{f} / H_{f}=0.89$ to 0.27$)$, and $\sqrt{\overline{v^{\prime 2}}} /\left(U_{1}-U_{2}\right)$ and $\sqrt{\overline{u^{\prime} v^{\prime}}} /\left(U_{1}-U_{2}\right)$, on the other hand, which decrease when approaching the bed. This could be explained 

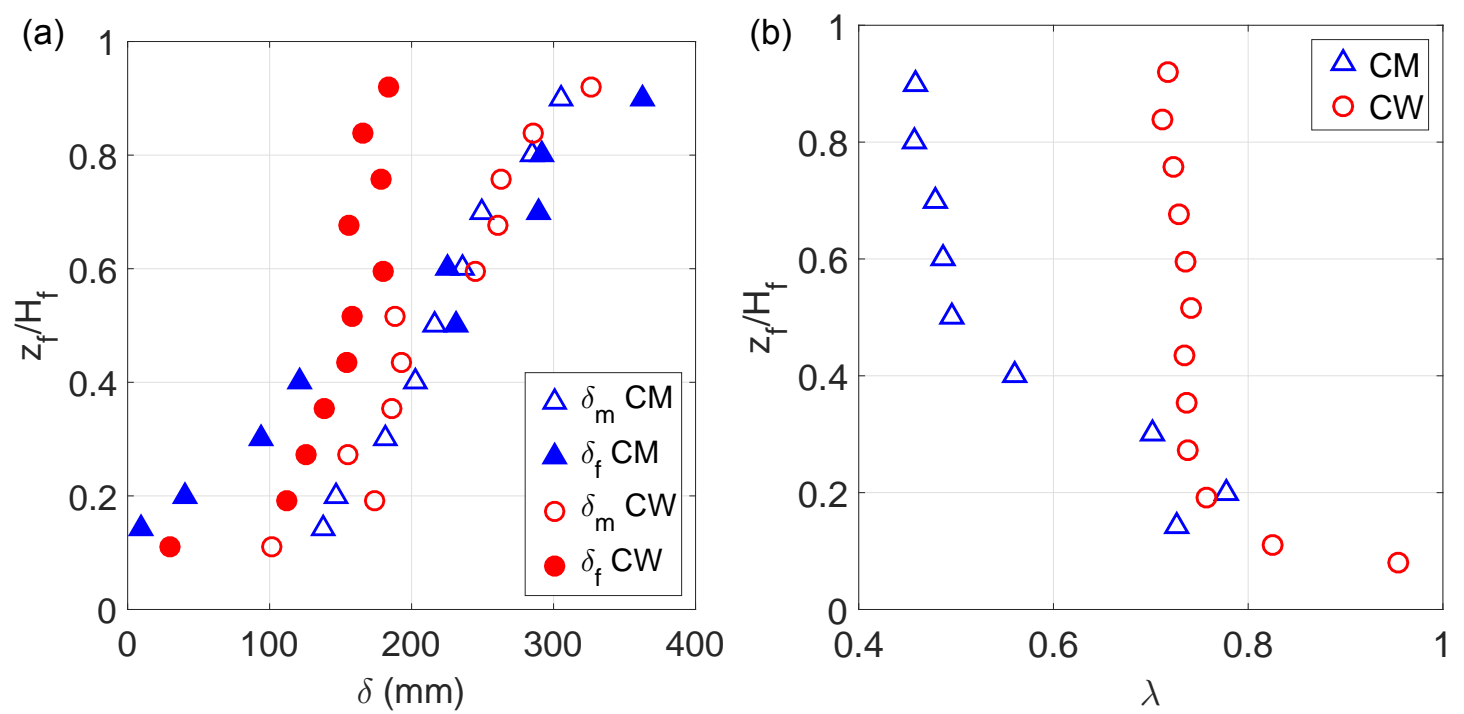

Fig. 8: Vertical variation in (a) main channel and floodplain mixing layer widths $\delta_{m}$ and $\delta_{f}$ and (b) normalised velocity difference $\lambda$ for test case CM (at $x=15.05 \mathrm{~m}$ ) and test case CW (at $x=11.76 \mathrm{~m})$.

as follows. Quantity $\overline{u^{\prime 2}}$ increases near the bed under the effect of bed-induced turbulence. The influence of bed-induced turbulence is less intense on $\overline{v^{\prime 2}}$ : according to Nezu and Nakagawa (1993) $\sqrt{\overline{u^{\prime 2}}} / U^{*}=2.30$ and $\sqrt{\overline{v^{\prime 2}}} / U^{*}=1.63$ at the bed level, where $U^{*}$ is the shear velocity. On the other hand, the bed-induced turbulence practically does not contribute to $\overline{u^{\prime} v^{\prime}}$. Therefore the dominant contribution to $\overline{v^{\prime 2}}$ and $\overline{u^{\prime} v^{\prime}}$ for $-1<\left(y-y_{I P}\right) / \delta_{i}<0$ is the mixing layer turbulence, which can be assumed to decrease near the bed, because the small-scale 3D bed turbulence tends to disorganize the large 2D turbulent coherent structures, compared to the upper water column. Besides, we note that when moving away from the interface towards the floodplain, i.e. $\left(y-y_{I P}\right) / \delta_{i}<-1$, the levels of $\sqrt{\overline{u^{\prime 2}}} /\left(U_{1}-U_{2}\right)$ and $\sqrt{\overline{v^{\prime 2}}} /\left(U_{1}-U_{2}\right)$ remain high at $z_{f} / H_{f}=0.27$, compared to the profiles at $z_{f} / H_{f}=0.50$ and 0.89 upper in the water column. This is due to the contribution of bed turbulence in the overall turbulence. The ratio $\sqrt{\overline{u^{\prime 2}}} / \sqrt{\overline{v^{\prime 2}}}$ is about 2 , which is higher but on the same order of magnitude as that given by Nezu and Nakagawa (1993), i.e.1.4.

\section{Effects of secondary currents and lateral Reynolds stresses in the main channel}

5.1 Cross-sectional distribution of longitudinal velocity

Figure 10 shows cross-sectional distribution $U(y, z)$, $V(y, z)$ and $-\rho \overline{u^{\prime} v^{\prime}}(y, z)$ in the main channel at $x=$
$15.05 \mathrm{~m}$ for test case CM (left charts) and at $x=$ $12.99 \mathrm{~m}$ for test case CW (right charts).

In the presence of grassed floodplains (test case $\mathrm{CM}), U(y, z)$ is characterized by two quasi-symmetric high velocity regions, separated by a velocity dip at $y_{\text {centre }}=1500 \mathrm{~mm}$, the centre of the channel (Fig. 10a). The two symmetric velocity peaks are located at $z / H_{m} \approx 0.73$ and at about $\left(y-y_{\text {centre }}\right) / B_{m}= \pm 0.15$. In contrast, for the flow with wooded floodplains CW (Fig. 10d), the contour-lines of $U$ are concentrically distributed around a unique velocity peak, which is located at $y_{\text {centre }}=1500 \mathrm{~mm}$ and at $z / H_{m} \approx 0.54$. The contour-lines of $U$ suggest that the distribution of longitudinal momentum is influenced by both turbulent mixing and secondary current cells.

The region $\left|\rho \overline{u^{\prime} v^{\prime}}\right|>0.7 \mathrm{~Pa}$ is depicted in Figs. 10c and $10 \mathrm{f}$ by red dotted lines. This region is more extended for test case CW than for test case CM. Therefore the turbulent diffusion of low momentum fluid from the floodplain to the main channel is more significant with emergent cylinders than with bed roughness only. As a result, the contour-lines of $U$ are more curved towards the main channel centreline for test case CW (Fig.10d) than for test case CM (Fig.10a).

The vertical mean velocities $W$ could not have been reliably measured with the ADV probe. Nevertheless, from the distribution of the lateral mean velocity $V(y, z)$, shown in Figs. 10b and 10e, we can infer the presence of six secondary current cells for test case CM (depicted in Fig. 10a with black arrows) and of four cells for test case CW (Fig. 10d), symmetrically distributed about $y_{\text {centre }}=1500 \mathrm{~mm}$. 
(a)
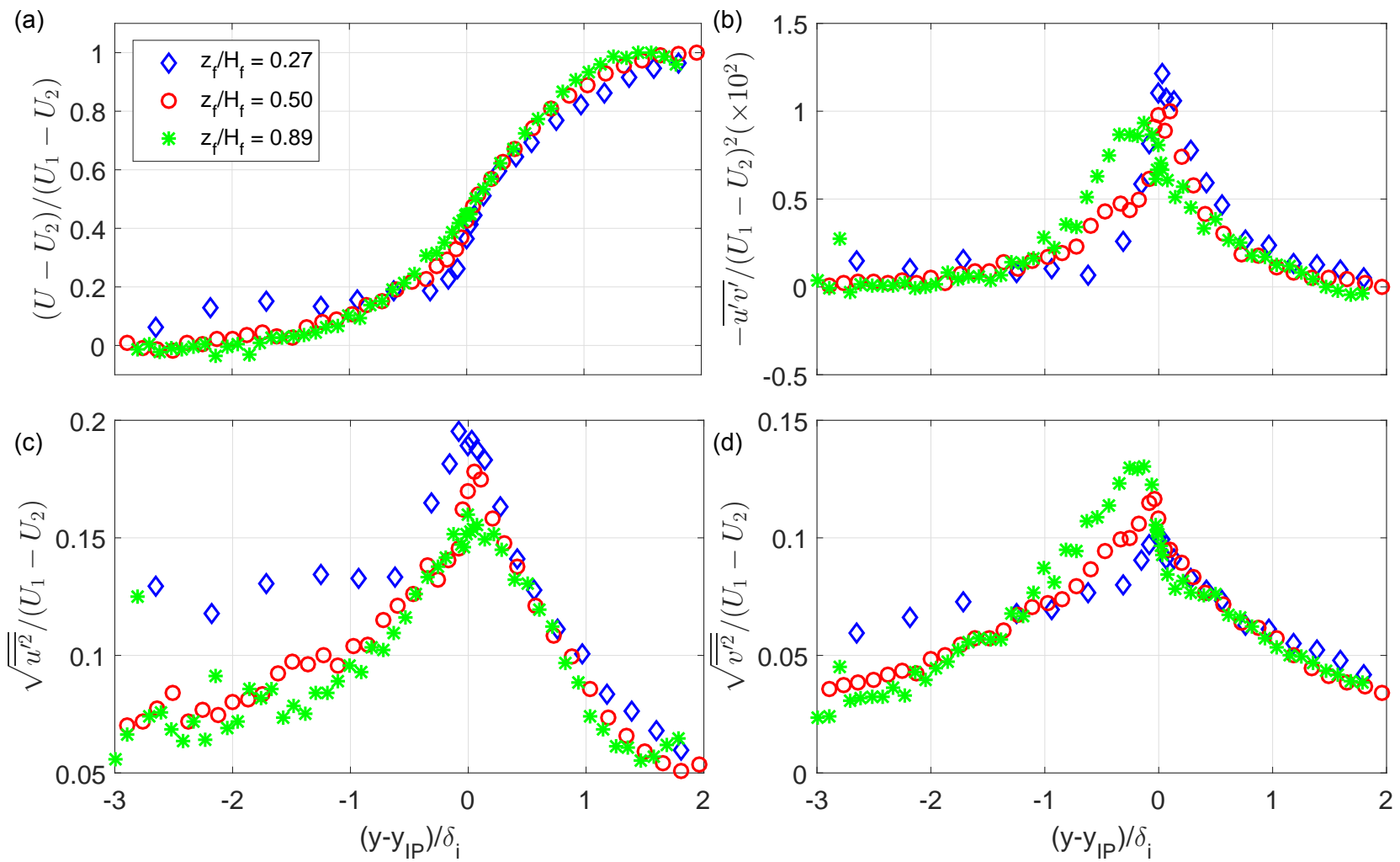

(d)

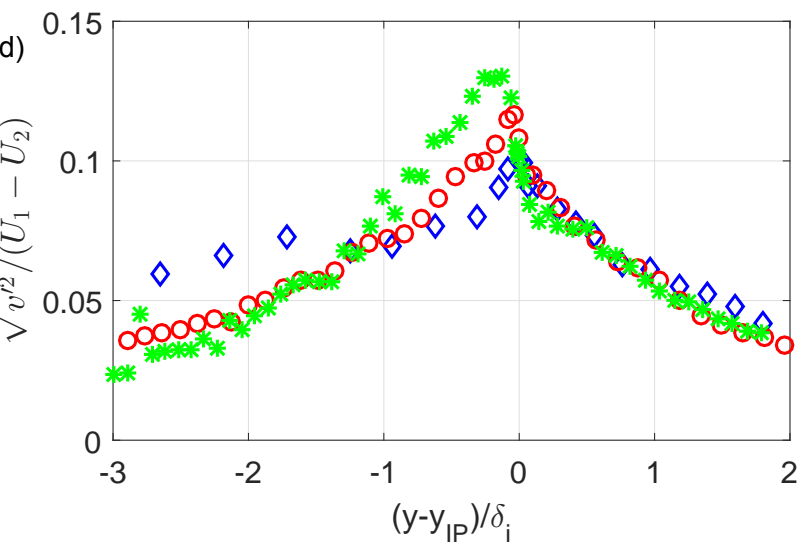

Fig. 9: Lateral profiles of normalised (a) mean longitudinal velocity, (b) lateral Reynolds stress, (c) longitudinal turbulence intensity and (d) lateral turbulence intensity at $x=13.15 \mathrm{~m}$ and at three different elevations for test case CM. Width $\delta_{i}=\delta_{f}$ on the floodplain side and $\delta_{i}=\delta_{m}$ on the main channel side.

The upper cell close to the interface for test case CM (cell 1) is characteristic of compound channel flows (Tominaga and Nezu, 1991) and is commonly called main channel vortex. This cell is usually associated with a counter-rotating cell on the floodplain side, the floodplain vortex, which is not observable with the present data. The cell close to the bottom (cell 2) has also been observed by Tominaga and Nezu (1991) for relative depths $D_{r}$ between 0.25 and 0.5 . A third cell (cell 3 ) is observed near the main channel centreline. This cell is not directly related to the compound channel geometry. It advects slow fluid from the bottom region up to the free-surface and generates a velocity dip at the main channel centreline.

Only cells 1 and 2 are observed for test case CW. They advect slow fluid from the interface region and from the lower main channel corner towards the main channel centreline and induce a concentric contour-line pattern. The absence of cell 3 is consistent with the absence of velocity dip at the centreline. When comparing the lateral velocity vectors in Figs. 10b and 10e, it appears that the secondary current magnitude is globally higher with emergent rigid cylinders over the floodplain than with bed roughness only.

Cell 3 for test case CM spans the entire flow depth, a characteristic that is typical for secondary cells in flat straight channels (Blanckaert et al, 2010). The widthto-height ratio of cells 1 and 2 taken together is 2 for test case CM and 2.3 for test case $\mathrm{CW}$, therefore relatively close. Assuming that the width-to-height ratio of cells $1+2$ is a constant for the present channel geometry and as the water depth is higher for test case CW than for test case CM, the absence of cell 3 for test case CW could then only be due to lack of space, which prevents the development of a couple of two symmetric cells near the channel centreline.

For compound channel flows with relative depths $D r=0.25$ and 0.5, Tominaga and Nezu (1991) found the width-to-height ratio of cells $1+2$ to be of the order of 1 with both smooth and rough floodplains. Similarly to the present study, the width-to-height ratio of cells $1+2$ does not depend on the floodplain roughness. However, this ratio is of the order of 2 in the present study, though Reynolds and Froude numbers are similar. It suggests that this ratio does depend on other factors 


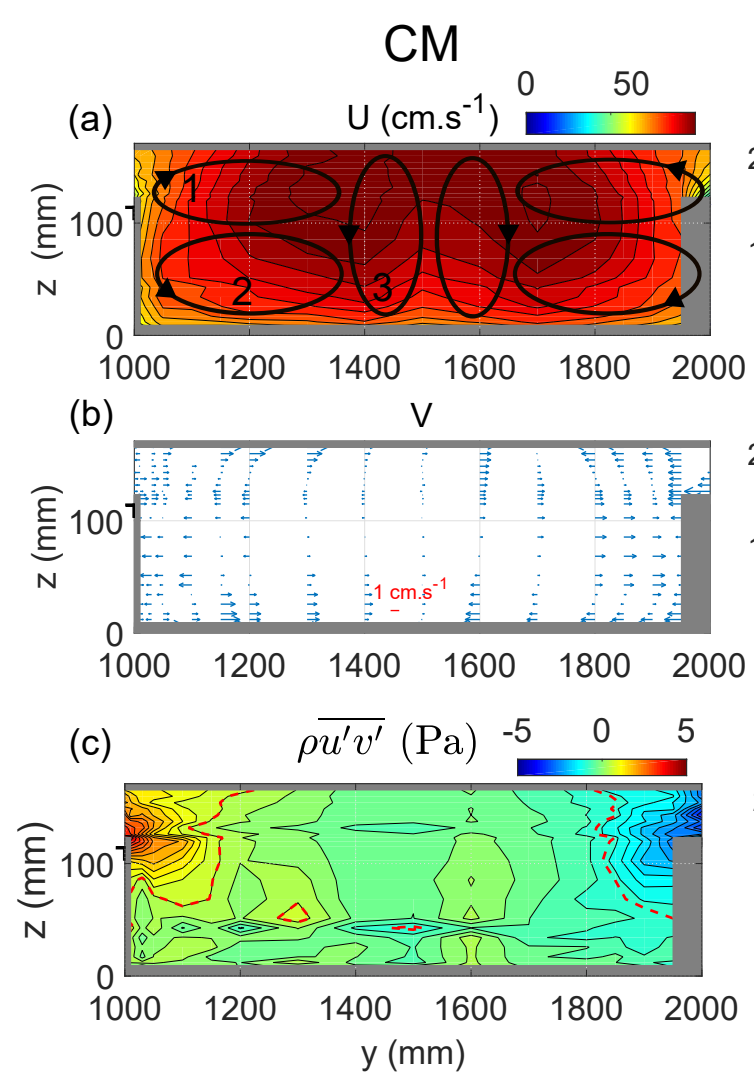

\section{CW}

(d)

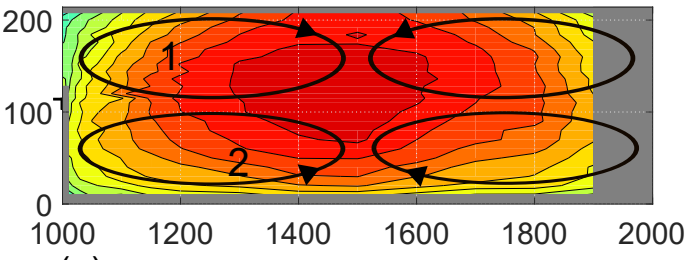

(e)

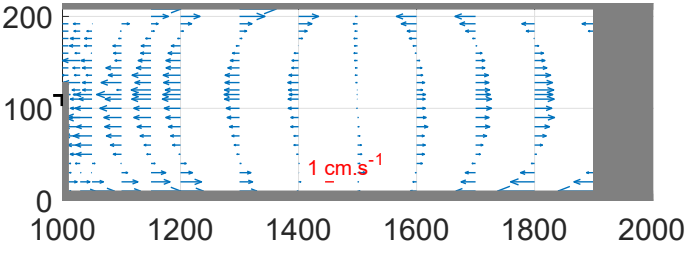

(f)

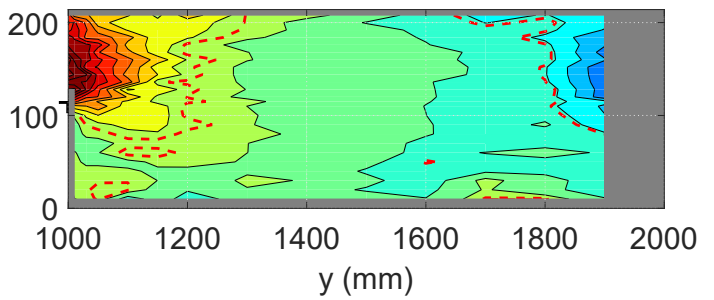

Fig. 10: Cross-sectional distribution of longitudinal mean velocity $U$, lateral mean velocity $V$ and lateral Reynolds stresses $-\rho \overline{u^{\prime} v^{\prime}}$ in the main channel (a-c) for test case CM at $x=15.05 \mathrm{~m}$ and (d-f) for test case CW at $x=12.99 \mathrm{~m}$. The red dotted lines in charts $\mathrm{c}$ and $\mathrm{f}$ are the contour-lines $+0.7 \mathrm{~Pa}$ and $-0.7 \mathrm{~Pa}$. Black arrows in charts a and $\mathrm{d}$ depict secondary current cells.

that could be the geometry of the compound channel (ratio $B_{m} / H_{m}$, symmetric or asymmetric channel).

\subsection{Lateral exchange of longitudinal momentum}

The time-averaged and depth-averaged lateral exchange of longitudinal momentum reads (Proust et al, 2013):

$\rho \overline{(u v)_{d}}=\rho\left(\overline{u^{\prime} v^{\prime}}\right)_{d}+\rho\left(U\left(V-V_{d}\right)\right)_{d}+\rho U_{d} V_{d}$

where subscript $d$ refers to depth-averaging. In the case of longitudinal uniformity, the depth-averaged lateral velocity $V_{d}$ is equal to zero; thus the lateral exchange of momentum $\rho \overline{(u v)_{d}}$ is driven by lateral Reynolds stresses and secondary currents only (first and second terms on the right-hand side of Eq. 3). Figure 11 shows the lateral distribution of these two terms in the main channel. The contributions of turbulent mixing and secondary currents to the lateral exchange of momentum are globally of the same order of magnitude. However, at the main channel/floodplain interface $y=1000 \mathrm{~mm}$, while the turbulent exchange is maximum, the momentum flux by the secondary currents vanishes. Thus, secondary currents only redistribute longitudinal momentum within the main channel and do not contribute significantly to the momentum exchange between the main channel and floodplains. This result is consistent with the previous analysis of Van Prooijen et al (2005).

The shape of $\rho\left(U\left(V-V_{d}\right)\right)_{d}(y)$ is similar for both test cases (fit with a fifth order polynomial function in Fig. 11b) although the cross-sectional distribution of the secondary current cells is very different (Fig.10). In particular, the lateral positions of the extremum values of $\rho\left(U\left(V-V_{d}\right)\right)_{d}$ are the same $(y=1200$ and $1800 \mathrm{~mm})$. The secondary currents advect momentum in the same direction as the lateral Reynolds stresses.

\subsection{Depth-averaged force balance}

The depth-averaged momentum equation in the main channel for a steady uniform flow reads (see e.g. Shiono 

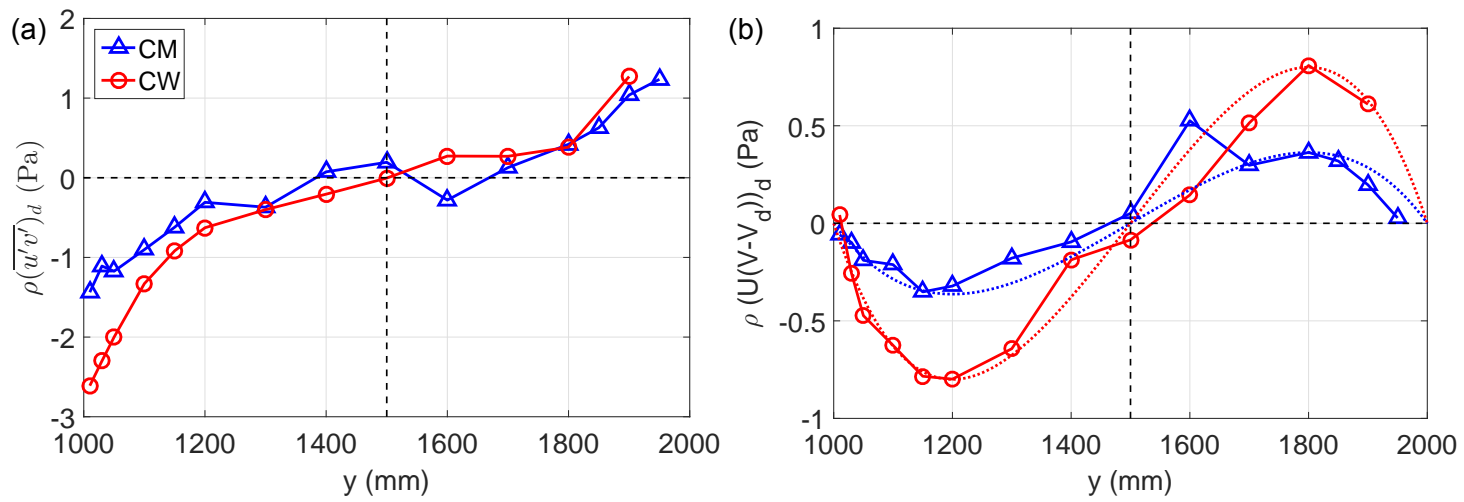

Fig. 11: Depth-averaged lateral exchange of momentum by (a) the lateral Reynolds stresses and (b) the secondary currents in the main channel for test cases CM and CW. Dotted lines in chart b are polynomial interpolations of degree 5 .

and Knight, 1991):

$S_{0}=\frac{1}{g H_{m}} \frac{\partial H_{m}(U V)_{d}}{\partial y}+\frac{1}{g H_{m}} \frac{\partial H_{m}\left(\overline{u^{\prime} v^{\prime}}\right) d}{\partial y}+\frac{\tau_{b}}{\rho g H_{m}}$

The three terms on the right-hand side of Eq. 4 relate to secondary currents, lateral Reynolds shear stress and bed friction, respectively. They were calculated from the experimental data and are plotted in Fig. 12 for both flow cases, after normalisation by $S_{0}$. The sum of the three contributions in Fig. 12 should therefore be equal to 1 , which is approximatively the case. Note that the second term on the right-hand side of Eq. 4 also takes into account the friction on the side walls. Bed shear stress (and lateral shear on the side walls) was calculated using the logarithmic law applied to the vertical (and horizontal) velocity profiles in the near wall region.

In the region $1000<y<1200 \mathrm{~mm}$, the force due to secondary currents tends to accelerate the flow and is therefore opposite to the force due to lateral Reynolds stresses (lateral Reynolds stresses are partly counterbalanced by the secondary current force). Both forces are of the same order of magnitude $(\mathrm{CM})$ or even one order of magnitude higher $(\mathrm{CW})$ than the bed friction force. In the region $1200<y<1500 \mathrm{~mm}$, both forces are resistant and lower than the bed friction.

To conclude on secondary currents, they advect longitudinal momentum from the main channel centreline towards the interface region (Fig. 11b), this lateral momentum flux being maximum at $y=1200 \mathrm{~mm}$ (when considering the right-half main channel) and vanishing at the interface with the floodplain. Secondary currents exert a driving force on the fluid (on an infinitesimal volume of width $d y$ ) in the region $1000<y<1200 \mathrm{~mm}$ (Fig. 12), that is opposite the lateral Reynolds stresses and of the same order of magnitude, and exert a resistance force on the fluid in the region $1200<y<$ $1500 \mathrm{~mm}$, that is of the same sign and of the same order of magnitude as the lateral Reynolds stresses.

\section{Coherent structures}

\subsection{Definitions and procedure}

The time series of longitudinal and lateral velocities feature large quasi-periodic oscillations in the interface region, as illustrated in Fig. 13a. These oscillations are the signature of coherent structures that are generated by the mixing layer. Quantities $u$ and $v$ are in opposite phase, so that these coherent events can be considered as an alternate succession of large sweeps $\left(u^{\prime}>0, v^{\prime}<\right.$ 0 , when considering the mixing layer at the right-hand interface) and ejections $\left(u^{\prime}<0, v^{\prime}>0\right.$, idem).

The two-point space-time correlation function is defined by (Shaw et al, 1995):

$R_{i k}^{(j)}\left(\xi_{j}, \tau\right)=\frac{\overline{u_{i}^{\prime}\left(x_{j}, t\right) u_{k}^{\prime}\left(x_{j}+\xi_{j}, t+\tau\right)}}{\left(\overline{u_{i}^{\prime 2}}\left(x_{j}, t\right) \overline{u_{k}^{\prime 2}}\left(x_{j}+\xi_{j}, t\right)\right)^{1 / 2}}$

where $\xi_{j}$ and $\tau$ are the spatial and time lags, $i, j, k \in$ $\{1,2,3\},\left(x_{1}, x_{2}, x_{3}\right)=(x, y, z)$ and $\left(u_{1}, u_{2}, u_{3}\right)=$ $(u, v, w)$.

The Eulerian integral time scales of the longitudinal and lateral velocity fluctuations $\tau_{11}$ and $\tau_{22}$ are calculated from the autocorrelation functions $R_{11}^{(-)}(0, \tau)$ and $R_{22}^{(-)}(0, \tau)$, respectively, and taken as four times the first zero-crossing, as sketched in Fig. 13b. Indeed, the first zero crossing of $R_{i i}^{(-)}(0, \tau)$ corresponds to a quarter period for a periodic signal. 

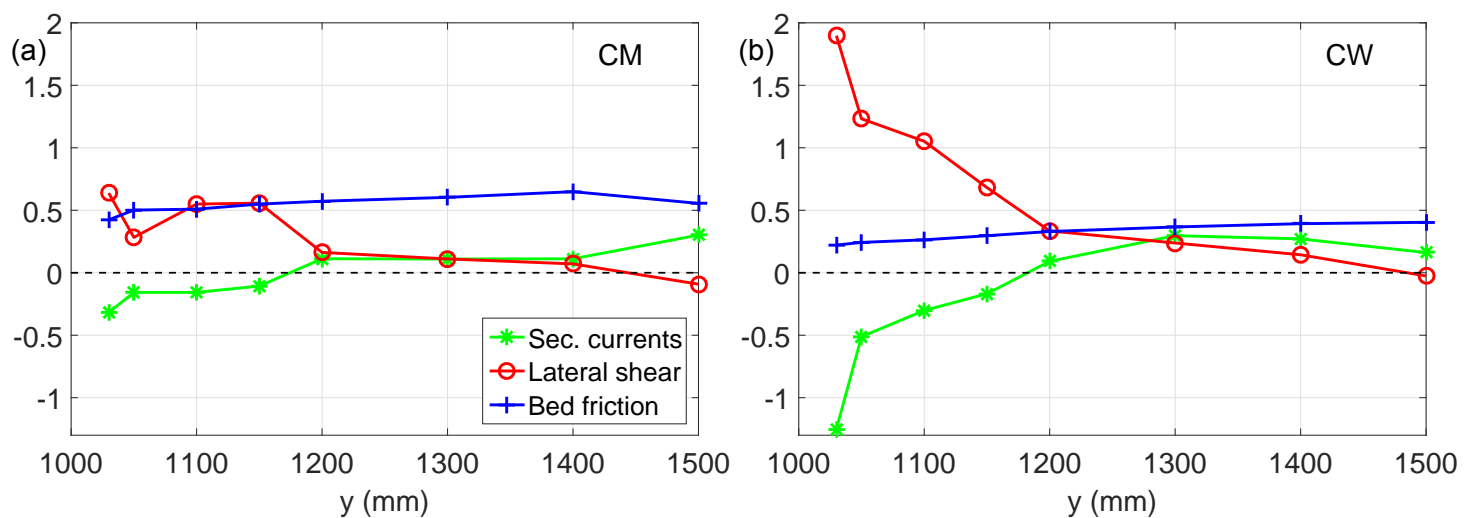

Fig. 12: Depth-averaged momentum balance in the main channel for (a) test case CM and (b) test case CW according to Eq. 4. The three terms on the right-hand side of Eq. 4 have been normalised by bottom slope $S_{0}$.
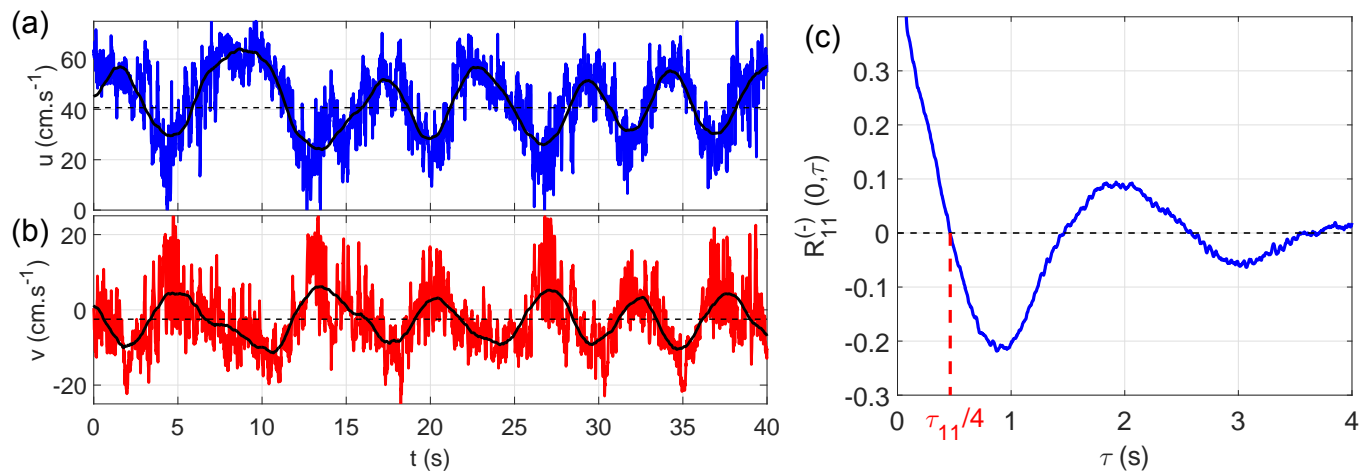

Fig. 13: Time series of (a) longitudinal and (b) lateral velocities for test case CW (measurement at $x=15.68 \mathrm{~m}$, $y=1000 \mathrm{~mm}, z_{f} / H_{f}=0.52$ ). The red and blue lines are the raw signals, the black lines are the low-pass-filtered signals and the dashed lines are the mean value of the raw signal. (b) Autocorrelation function of longitudinal velocity fluctuations for test case $\mathrm{CW}$ (measurement at $x=11.12 \mathrm{~m}, y=1000 \mathrm{~mm}$ and $z_{f} / H_{f}=0.78$ ) and definition of the integral time scale $\tau_{11}$.

Furthermore, for a given space lag $\xi_{j}$, the maximum correlation $R_{i i \text { max }}^{(j)}$ is the correlation at the time lag $\tau$ that maximises $R_{i i}^{(j)}$ :

$R_{i i, \max }^{(j)}\left(\xi_{j}\right)=\max _{\tau} R_{i i}^{(j)}\left(\xi_{j}, \tau\right)$

The autocorrelation functions $R_{11}^{(-)}$and $R_{22}^{(-)}$were calculated with the raw time series. In order to remove the influence of the small scale fluctuations, which are uncorrelated even for small spatial lags, the two-point space-time correlations were calculated with low-pass filtered signals, using a Savitzky-Golay smoothing filter (Matlab function 'sgolayfilt'). Low-pass-filtered velocity signals are shown in Fig. 13a for test case CW (black lines).

\subsection{Phase velocity}

If the coherent velocity fluctuations are considered as waves that propagate downstream, one can define the phase velocity of the coherent fluctuations of the longitudinal and lateral velocity, $U_{11}$ and $U_{22}$. Using the Taylor hypothesis, the Eulerian integral time scales can then be converted into longitudinal Eulerian integral length scales with the equation $L_{i i}^{(1)}=U_{i i} \tau_{i i}$.

The experimental determination of phase velocity $U_{i i}$ is often a difficult task. Zaman and Hussain (1981) tested different assumptions for approximating phase velocity (local instantaneous velocity, local mean velocity, etc.) and found that the best results were obtained by taking a constant velocity across the entire coherent structure, equal to the mean velocity at the centre of the structure. In our study, a direct estimation of the phase velocities was undertaken at some locations, in 
order to assess the best choice for approximating $U_{i i}$. Phase velocity was evaluated by tracking the coherent events between two points separated by a longitudinal spatial lag (space-time correlation in the longitudinal direction). The coherent events were detected using the local velocity maximum in the low-pass filtered time series; only coherent events that could be clearly recognized were selected. Phase velocities were obtained by averaging about 100 selected coherent events.

Phase velocities $U_{11}$ and $U_{22}$ at the interface $(y=$ $1000 \mathrm{~mm}$ ) and at elevation $z_{f}=H_{f} / 2$ for both test cases CM and CW are presented in Table 3 and are compared with the local mean longitudinal velocity and with the depth averaged mean longitudinal velocity. Phase velocities $U_{11}$ and $U_{22}$ are identical within the measurement accuracy for both test cases. The depthaveraged velocity at the interface seems to give a rather good estimate of the phase velocities. The local mean velocity is not adapted, especially for test case CM, for which it would overestimate by $15 \%$ the measured phase velocity.

In the following, when using the Taylor hypothesis, the phase velocity is taken as the depth-averaged longitudinal velocity at the interface and is assumed to be constant over the depth, following the recommendations of Zaman and Hussain (1981). No distinction is made between the phase velocity of longitudinal and lateral velocity fluctuations.

Table 3: Estimation of the phase velocities of longitudinal and lateral velocity fluctuations $U_{11}$ and $U_{22}$ at the interface $(y=1000 \mathrm{~mm})$ and at $z_{f}=H_{f} / 2$ for test cases CM $(x=14.80 \mathrm{~m})$ and CW $(x=14.55 \mathrm{~m})$. Comparison with depth averaged mean longitudinal velocity $U_{d}$ and the local punctual time-averaged velocity $U_{\text {local }}$.

\begin{tabular}{ccccc}
\hline Test case & $U_{11}$ & $U_{22}$ & $U_{d}$ & $U_{\text {local }}$ \\
\hline $\mathrm{CM}$ & $45.5 \pm 3$ & $46.5 \pm 3$ & $48 \pm 1$ & $53 \pm 1$ \\
$\mathrm{CW}$ & $39 \pm 3$ & $36 \pm 3$ & $40.5 \pm 1$ & $39 \pm 1$ \\
\hline
\end{tabular}

\subsection{Longitudinal evolution}

Figure 14a shows longitudinal profiles $\tau_{11}(x)$ and $\tau_{22}(x)$ at the interface $y=1000 \mathrm{~mm}$ and at $z_{f} / H_{f}=0.20$ for test case $\mathrm{CM}$ and $z_{f} / H_{f}=0.60$ for test case $\mathrm{CW}$. Time scales $\tau_{i i}$, and therefore the longitudinal size of the coherent structures, grow approximately linear up to the channel end for test case CM. The coherent structure size is fairly constant for test case CW after $x \approx 10 \mathrm{~m}$. For both flows $\tau_{11}$ is higher than $\tau_{22}$, which implies, by using the Taylor hypothesis, that the coherent structures related to $U$ are longer than those related to $V$. The ratio $\tau_{11} / \tau_{22}$ is about 1.4 for $\mathrm{CM}$ and 1.25 for $\mathrm{CW}$, which is very close to the values found by Nikora et al (2007), i.e. between 1.19 and 1.37, for large-scale coherent structures in super- and sub-critical shallow openchannel flows. The present results suggest that coherent structures are not characterised by a single frequency but at least by two different frequencies for the longitudinal and lateral velocity components.

Figure 14b shows the longitudinal length scales $L_{11}^{(1)}$ and $L_{22}^{(1)}$ scaled by the total mixing layer width $\delta_{t o t}=\delta_{m}+\delta_{f}$. It appears that the longitudinal size of the coherent structures is approximatively proportional to the total mixing layer width, especially far from the channel ends, between $x=5$ and $x=15 \mathrm{~m}$. A similar result was obtained for plane mixing layers by Brown and Roshko (1974), who showed that the mean distance $L_{C S}$ between two successive coherent structures is proportional to the vorticity width of the mixing layer, defined by $\delta_{\omega}=\left(U_{1}-U_{2}\right) /(d U / d y)_{\max }$, and they estimated $L_{C S} / \delta_{\omega} \approx 2.9$. The present results show that the ratios $L_{i i}^{(1)} / \delta_{\text {tot }}$ depend on floodplain occupation and are higher for the compound channel flow with wooded floodplains than with grassed floodplains.

According to Ho and Huerre (1984), the coherent structures of the plane mixing layer are generated with a preferred mode, which is characterised by the Strouhal number $S t=f \theta / U_{0}$, where $f$ is the passage frequency of the coherent structures and $\theta$ is the momentum thickness of the mixing layer, defined by:

$\theta=\int_{-\infty}^{+\infty}\left(\frac{1}{4}-\left(\frac{U-U_{0}}{U_{1}-U_{2}}\right)^{2}\right) d y$

The natural Strouhal number of the plane mixing layer is $S t=0.032$ for sufficiently low free stream velocities. If the free stream velocities are such that the initial boundary layers at the splitter plates are turbulent, the Strouhal number of the plane mixing layer is increased to $S t=0.044-0.048$ (Ho and Huerre, 1984). Figure 15 shows the longitudinal evolution of the Strouhal number for test case CM. The Strouhal number is calculated either with the passage frequency of the $u$ fluctuations $\theta /\left(\tau_{11} U_{0}\right)$ or with the passage frequency of the $v$-fluctuations $\theta /\left(\tau_{22} U_{0}\right)$. The momentum thickness, which measures the difference in momentum with respect to a potential flow formed by two parallel streams of uniform velocities $U_{1}$ and $U_{2}$, is calculated with a more general definition, adapted for an asymmetric mixing layer:

$\theta=\frac{\int_{-\infty}^{y_{I P}}\left(U_{2}^{2}-U^{2}\right) d y+\int_{y_{I P}}^{\infty}\left(U_{1}^{2}-U^{2}\right) d y}{\left(U_{1}-U_{2}\right)^{2}}$. 

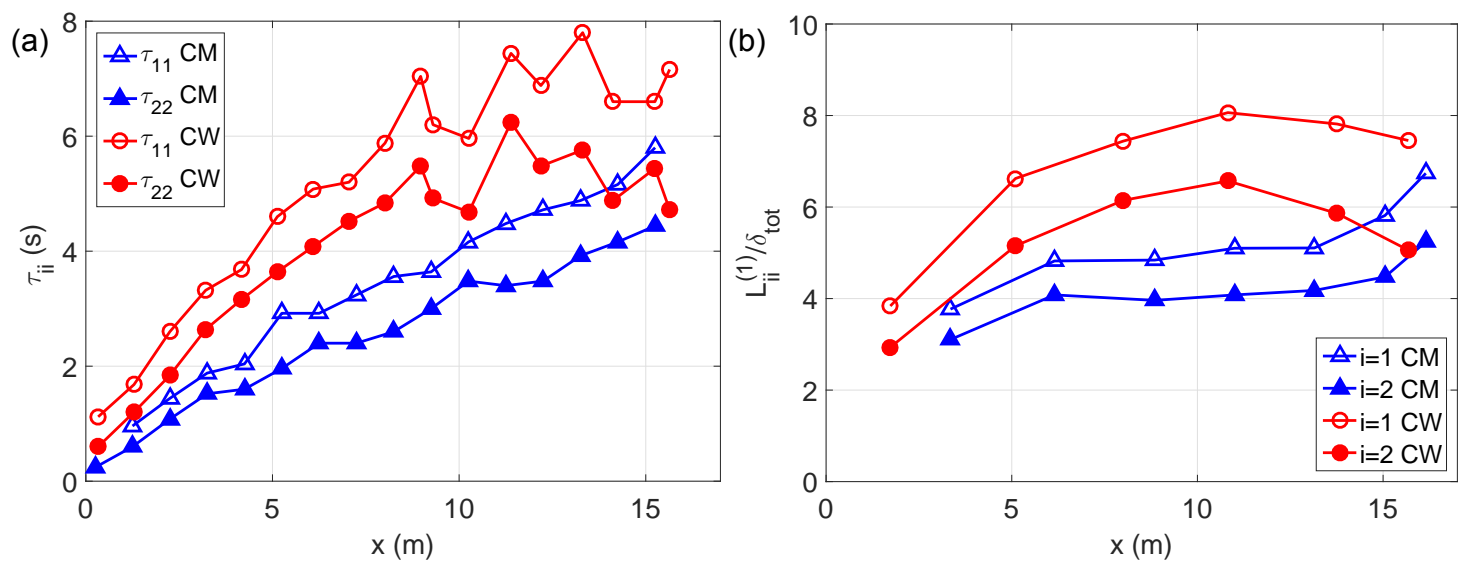

Fig. 14: (a) Longitudinal evolution along the interface $(y=1000 \mathrm{~mm})$ of the integral time scales $\tau_{11}$ and $\tau_{22}$ for test cases $\mathrm{CM}\left(\right.$ at $\left.z_{f} / H_{f}=0.20\right)$ and $\mathrm{CW}\left(\right.$ at $\left.z_{f} / H_{f}=0.60\right)$. (b) Integral length scales $L_{11}^{(1)}$ and $L_{22}^{(1)}$ normalised by the total mixing layer width.

Figure 15 shows that the Strouhal number is not constant when going downstream but stabilises around $x \approx$ $12 \mathrm{~m}$. The value $\theta /\left(\tau_{11} U_{0}\right)$ converges to 0.034 , i.e. close to the value of the plane mixing layer. The Strouhal number associated with the $v$-fluctuations $\theta /\left(\tau_{22} U_{0}\right)$ converges to a somewhat higher value of 0.044 .

For test case $\mathrm{CW}$ the computation of $\theta$ is biased by the cylinder wakes that cause velocity dips in the lateral profile of $U$. In the case of a mixing layer caused by an array of emergent cylinders on the low speed side, White and Nepf (2007) found the passage frequency of the coherent structures to be equal to the Strouhal number value of the plane mixing layer $S t=0.032$ (they computed $\theta$ based on the lateral profile of the spatially averaged longitudinal velocity, which smooths the cylinder wakes).

It can therefore be concluded that the Strouhal number value $S t \approx 0.032$ for mixing layers is quite universal, irrespective of the different mixing layer types. However, the present study points out that the Strouhal numbers associated with the $u$-and $v$-fluctuations are a priori different, and that for the compound channel mixing layer, the Strouhal number is not constant in the near-field region.

\subsection{Vertical extension}

Figure 16a shows vertical profiles $\tau_{11}(z)$ and $\tau_{22}(z)$ for test cases CM and CW. Time scales $\tau_{11}$ and $\tau_{22}$ are nearly constant across the water column (a decrease of about $10 \%$ is observed from the floodplain bottom to the free surface). Assuming a constant phase velocity over the depth, as stated in Section 6.2, it follows that

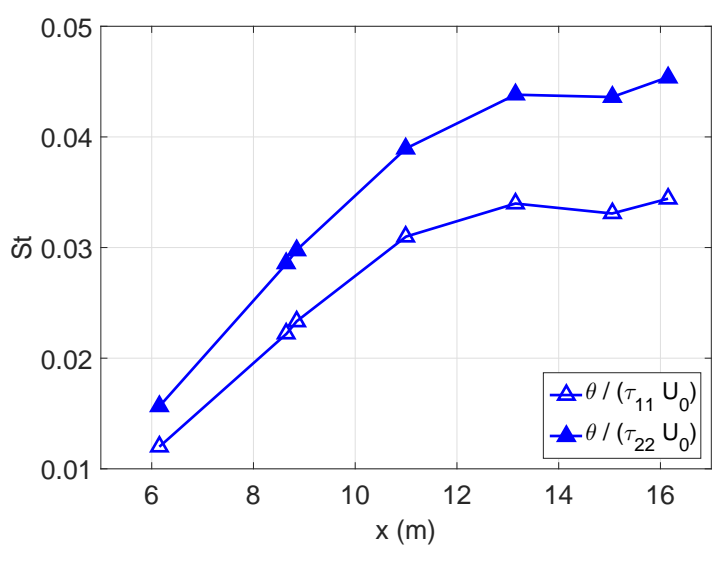

Fig. 15: Longitudinal evolution of the Strouhal number associated with the mixing layer of test case CM. Measurement at $z_{f} / H_{f}=0.5$.

the integral length scales $L_{11}^{(1)}$ and $L_{22}^{(1)}$, and therefore the longitudinal size of the coherent structures, are also constant across the water column. The ratio $\tau_{11} / \tau_{22}$ is constant over the depth and is about 1.3 for both test cases CM and CW (see Section 6.3).

In order to investigate the vertical extent of the coherent structures, two-point space-time correlations were carried out in the vertical direction. The reference probe was kept constant near the free surface (at position $\left(x^{r}, y^{r}, z^{r}\right)=(13.82 \mathrm{~m}, 1000 \mathrm{~mm}, 166 \mathrm{~mm})$ for test case CM and $(13.76 \mathrm{~m}, 1000 \mathrm{~mm}, 192 \mathrm{~mm})$ for test case CW) and the moving probe was moved downwards. Figure $16 \mathrm{~b}$ shows the maximum correlation $R_{11, \max }^{(3)}(z)$ and $R_{22, \max }^{(3)}(z)$. A slight loss of correlation is observed when the two probes are moved away from each other, 
(a)

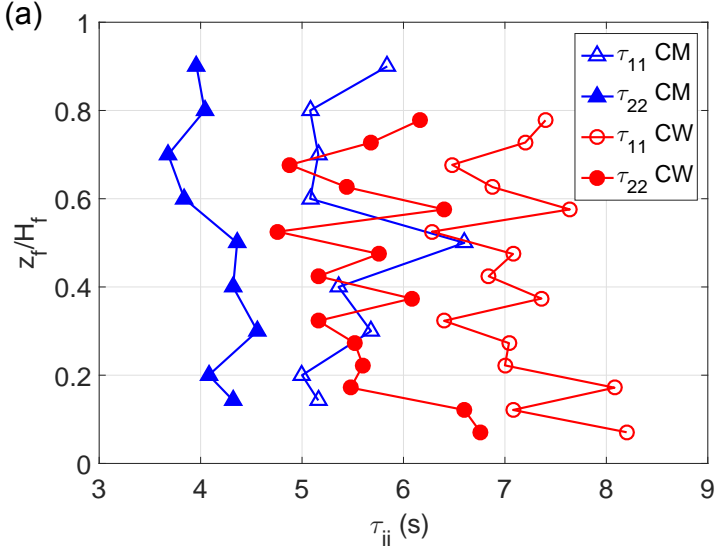

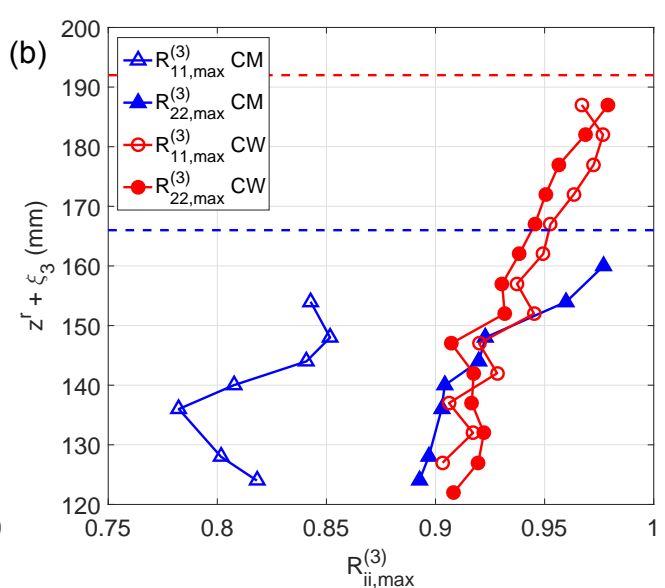

Fig. 16: (a) Vertical variation in the integral time scales $\tau_{i i}$ of the longitudinal and lateral velocity fluctuations for test cases CM (at $x=15.05 \mathrm{~m}$ ) and CW (at $x=13.76 \mathrm{~m}$ ) at the interface $(y=1000 \mathrm{~mm}$ ). (b) Maximum of correlation $R_{i i, \max }^{(3)}$ in the vertical direction for test case CM with reference probe at $\left(x^{r}=13.82 \mathrm{~m}, y^{r}=y_{\text {int }}\right.$, $\left.z^{r}=166 \mathrm{~mm}\right)$ and for test case $\mathrm{CW}$ with reference probe at $\left(x^{r}=13.76 \mathrm{~m}, y^{r}=y_{\text {int }}, z^{r}=192 \mathrm{~mm}\right)$; the vertical position of the reference probe is marked with dotted lines.

mainly for test case CM, but the maximum correlations for the two velocity components remains high $(>0.8)$. It suggests that the coherent structure forms a unique entity that spans the entire water column.

\section{Conclusion}

Two compound channel flows with floodplains covered with either a bed roughness (meadow-type vegetation) or an emergent cylinder array installed on a bed roughness (wood-type vegetation) were experimentally investigated. The two flows were developing downstream without reaching a uniform state, but were characterised by self-similarity. The main results are summarised below:

- To take into account the asymmetry of the compound channel mixing layer, two mixing layer widths on both sides of the mean velocity inflection point were defined: $\delta_{m}$ on the main channel side and $\delta_{f}$ on the floodplain side.

- Both the growth rate of the mixing layer and the stabilisation of its width differed on the main channel side and on the floodplain side. Width $\delta_{f}$ stabilised under the effect either of vertical flow confinement or of the presence of emergent roughness in the floodplain. The latter effect was stronger than the former. The wide main channel used in the present study prevented a stabilisation of $\delta_{m}$ by lateral flow confinement within the flume length. Therefore no state of longitudinal flow uniformity was observed.
- The compound channel mixing layer was self-similar in the longitudinal direction at a given elevation, when the flow quantities were normalised by the velocity difference across the mixing layer and the lateral coordinate was normalised by $\delta_{m}$ or $\delta_{f}$ on either side of the inflection point.

- The compound channel mixing layer was highly heterogeneous in the vertical direction, in particular the mixing layer width increased and the dimensionless velocity difference $\lambda$ decreased from the floodplain bottom to the free surface. The presence of emergent roughness elements in the floodplain homogenised the mixing layer in the vertical direction. The normalised lateral profiles of velocity and turbulence did not coincide at different elevations, notably on the floodplain side, because of an increasing effect of bed-induced turbulence on mixing layer turbulence from the free surface to the floodplain bottom.

- The present study confirmed that the contribution of secondary currents to the momentum exchange between floodplain and main channel is negligible (Van Prooijen et al, 2005). However, secondary currents played a major role in the redistribution of longitudinal momentum within the main channel and exerted a force on the fluid that was on the same order of magnitude as the lateral Reynolds stresses. Secondary currents were stronger with wooded floodplains than with grassed floodplains.

- Turbulent coherent structures in the form of a succession of large sweep and ejection events were ob- 
served in the compound channel mixing layer. The phase velocity of the coherent structures could be roughly approximated by the depth-averaged longitudinal velocity at the main channel/floodplain interface. The longitudinal length scale of the coherent structures scaled with the total mixing layer width. The longitudinal and lateral velocity fluctuations were characterised by different Strouhal number values. These Strouhal number values were comparable to those found for plane mixing layers $S t=0.032-0.044$. Spatial correlations of the velocity signals showed that coherent structures spanned the entire floodplain flow depth and had a constant length over the water column.

Acknowledgements The PhD grant of V. Dupuis was funded by Irstea and by the French National Research Agency (Flowres project, grant No. ANR-14-CE03-0010, https://flowres.irstea.fr/en/). The authors greatly thank Fabien Thollet and Alexis Buffet for their technical support.

\section{References}

Bell JH, Mehta RD (1990) Development of a twostream mixing layer from tripped and untripped boundary layers. AIAA journal 28(12):2034-2042

Bernal L, Roshko A (1986) Streamwise vortex structure in plane mixing layers. Journal of Fluid Mechanics 170:499-525

Blanckaert K, Duarte A, Schleiss A (2010) Influence of shallowness, bank inclination and bank roughness on the variability of flow patterns and boundary shear stress due to secondary currents in straight openchannels. Advances in Water Resources 33(9):10621074

Bousmar D, Riviere N, Proust S, Paquier A, Morel R, Zech Y (2005) Upstream discharge distribution in compound-channel flumes. Journal of Hydraulic Engineering 131(5):408-412

Brown GL, Roshko A (1974) On density effects and large structure in turbulent mixing layers. Journal of Fluid Mechanics 64(04):775-816

Chu VH, Babarutsi S (1988) Confinement and bedfriction effects in shallow turbulent mixing layers. Journal of hydraulic engineering 114(10):1257-1274

Dupuis V (2016) Experimental investigation of flows subjected to a longitudinal transition in hydraulic roughness in single and compound channels. $\mathrm{PhD}$ thesis, Université de Lyon

Dupuis V, Proust S, Berni C, Paquier A (2016) Combined effects of bed friction and emergent cylinder drag in open channel flow. Environmental Fluid Mechanics 16(6):1173-1193
Fernandes J (2013) Compound channel uniform and non-uniform flows with and without vegetation in the floodplain. PhD thesis, Ph. D. thesis Departamento de Engenharia Civil. Instituto Superior Técnico da Universidade Técnica de Lisboa

Fjortoft R (1950) Application of integral theorems in deriving criteria of stability for laminar flows and for the baroclinic circular vortex. Geofysiske Publikasjoner 17(6):1-52

Goring DG, Nikora VI (2002) Despiking acoustic Doppler velocimeter data. Journal of Hydraulic Engineering 128(1):117-126

Ho CM, Huerre P (1984) Perturbed free shear layers. Annual Review of Fluid Mechanics 16(1):365-422

Kara S, Stoesser T, Sturm TW (2012) Turbulence statistics in compound channels with deep and shallow overbank flows. Journal of Hydraulic Research 50(5):482-493

Knight D, Shiono K (1990) Turbulence measurements in a shear layer region of a compound channel. Journal of hydraulic research 28(2):175-196

Knight DW, Demetriou JD (1983) Flood plain and main channel flow interaction. Journal of Hydraulic Engineering 109(8):1073-1092

Kozioł A (2013) Three-dimensional turbulence intensity in a compound channel. Journal of Hydraulic Engineering 139(8):852-864

Liu D, Diplas P, Fairbanks J, Hodges C (2008) An experimental study of flow through rigid vegetation. Journal of Geophysical Research: Earth Surface (2003-2012) 113(F4)

Nezu I, Nakagawa H (1993) Turbulence in open channels. IAHR/AIRH Monograph Balkema, Rotterdam, The Netherlands

Nezu I, Nakayama T (1997) Space-time correlation structures of horizontal coherent vortices in compound open-channel flows by using particletracking velocimetry. Journal of Hydraulic Research 35(2):191-208

Nicollet G, Uan M (1979) Ecoulements permanents à surface libre en lits composés. La Houille Blanche 1979(1):21-30

Nikora V, Nokes R, Veale W, Davidson M, Jirka G (2007) Large-scale turbulent structure of uniform shallow free-surface flows. Environmental Fluid Mechanics $7(2): 159-172$

Olsen MG, Dutton JC (2002) Stochastic estimation of large structures in an incompressible mixing layer. AIAA journal 40(12):2431-2438

Oster D, Wygnanski I (1982) The forced mixing layer between parallel streams. Journal of Fluid Mechanics 123:91-130 
Pasche E, Rouvé G (1985) Overbank flow with vegetatively roughened flood plains. Journal of Hydraulic Engineering 111(9):1262-1278

Peltier Y (2011) Physical modelling of overbank flows with a groyne set on the floodplain. PhD thesis, Université de Lyon

Proust S, Fernandes JN, Peltier Y, Leal JB, Riviere N, Cardoso AH (2013) Turbulent non-uniform flows in straight compound open-channels. Journal of Hydraulic Research 51(6):656-667

Proust S, Fernandes JN, Leal JB, Riviere N, Peltier Y (accepted) Mixing layer and coherent structures in compound channel flows: effects of transverse flow, velocity ratio and vertical confinement. Water resources research

Sellin RHJ (1964) A laboratory investigation into the interaction between the flow in the channel of a river and that over its flood plain. La Houille Blanche 1964(7):793-802

Shaw R, Brunet Y, Finnigan J, Raupach M (1995) A wind tunnel study of air flow in waving wheat: twopoint velocity statistics. Boundary-Layer Meteorology $76(4): 349-376$

Shiono K, Knight DW (1991) Turbulent open-channel flows with variable depth across the channel. Journal of Fluid Mechanics 222:617-646

Stocchino A, Brocchini M (2010) Horizontal mixing of quasi-uniform straight compound channel flows. Journal of Fluid Mechanics 643:425-435

Tamai N, Asaeda T, Ikeda H (1986) Study on generation of periodical large surface eddies in a composite channel flow. Water Resources Research 22(7):11291138

Tominaga A, Nezu I (1991) Turbulent structure in compound open-channel flows. Journal of Hydraulic Engineering $117(1): 21-41$

Uijttewaal W, Booij R (2000) Effects of shallowness on the development of free-surface mixing layers. Physics of Fluids (1994-present) 12(2):392-402

Van Prooijen BC, Battjes JA, Uijttewaal WS (2005) Momentum exchange in straight uniform compound channel flow. Journal of hydraulic engineering 131(3):175-183

Vermaas D, Uijttewaal W, Hoitink A (2011) Lateral transfer of streamwise momentum caused by a roughness transition across a shallow channel. Water resources research $47(2)$

White BL, Nepf HM (2007) Shear instability and coherent structures in shallow flow adjacent to a porous layer. Journal of Fluid Mechanics 593:1-32

Wood D, Bradshaw P (1984) A turbulent mixing layer constrained by a solid surface. Part 2. Measurements in the wall-bounded flow. Journal of fluid mechanics
$139: 347-361$

Yule AJ (1972) Two-dimensional self-preserving turbulent mixing layers at different free stream velocity ratios. HM Stationery Office

Zaman K, Hussain A (1981) Taylor hypothesis and large-scale coherent structures. J Fluid Mech 112(2):379-396

Zong L, Nepf H (2010) Flow and deposition in and around a finite patch of vegetation. Geomorphology 116(3):363-372 